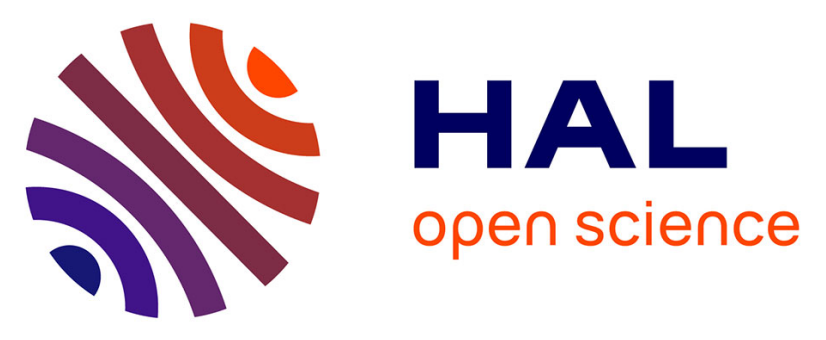

\title{
Impact of Changing the Core in Tetrapyrrolic Dendrimers Designed for Oxygen Sensitization: New Fluorescent Phthalocyanine-Based Dendrimers with High Two-Photon Absorption Cross-sections
}

Seifallah Abid, Sarra Ben Hassine, Zhipeng Sun, Nicolas Richy, Franck Camerel, Bassem Jamoussi, Mireille Blanchard-desce, Olivier Mongin, Frédéric Paul, Christine Paul-Roth

\section{- To cite this version:}

Seifallah Abid, Sarra Ben Hassine, Zhipeng Sun, Nicolas Richy, Franck Camerel, et al.. Impact of Changing the Core in Tetrapyrrolic Dendrimers Designed for Oxygen Sensitization: New Fluorescent Phthalocyanine-Based Dendrimers with High Two-Photon Absorption Cross-sections. Macromolecules, 2021, 54 (14), pp.6726-6744. 10.1021/acs.macromol.1c00830 . hal-03331154

\author{
HAL Id: hal-03331154 \\ https://hal.science/hal-03331154
}

Submitted on 15 Sep 2021

HAL is a multi-disciplinary open access archive for the deposit and dissemination of scientific research documents, whether they are published or not. The documents may come from teaching and research institutions in France or abroad, or from public or private research centers.
L'archive ouverte pluridisciplinaire HAL, est destinée au dépôt et à la diffusion de documents scientifiques de niveau recherche, publiés ou non, émanant des établissements d'enseignement et de recherche français ou étrangers, des laboratoires publics ou privés. 
Impact of changing the core in tetrapyrrolic dendrimers designed for oxygen sensitization: New fluorescent phthalocyanines-based dendrimers with high two-photon absorption cross-sections

Seifallah Abid, ${ }^{\text {a,b }}$ Sarra Ben Hassine, ${ }^{a, c}$ Zhipeng Sun, ${ }^{a}$ Nicolas Richy, ${ }^{a}$ Franck Camerel, ${ }^{a}$ Bassem Jamoussi, ${ }^{d}$ Mireille Blanchard-Desce, ${ }^{e}$ Olivier Mongin, ${ }^{a}$ Frédéric Paul, ${ }^{a}$ Christine O. PaulRoth, ${ }^{* a}$

Dedicated to Professor Kenneth RAYMOND for his stimulating work on phthalocyanine chemistry, and also its so positive energy

${ }^{a}$ Univ Rennes, INSA Rennes, CNRS, ISCR (Institut des Sciences Chimiques de Rennes) - UMR 6226, F-35000 Rennes, France

${ }^{b}$ Université de Carthage, Faculté des Sciences de Bizerte, Tunisie

${ }^{c}$ Faculté des Sciences de Tunis, Université de Tunis El Manar, Tunisie

${ }^{d}$ Department of Environmental Sciences, Faculty of Meteorology, Environment and Arid Land Agriculture, King Abdulaziz University, Jeddah, Saudi Arabia

${ }^{e}$ Univ. Bordeaux, Institut des Sciences Moléculaires (CNRS UMR 5255), 33405 Talence, France

*Corresponding author: christine.paul@univ-rennes1.fr or christine.paul@insa-rennes.fr tel: (+33) (0) 223236372

ABSTRACT: In the continuation of our sustained interest for porphyrin-based dendrimers and their use as luminescent photosensitizers for two-photon photodynamic therapy (2P-PDT), we wondered about the effect of changing the central porphyrin core for a phthalocyanine core in these macromolecular structures. Thus, related phthalocyanine-based dendrimers possessing up to sixteen conjugated 9,9-dibutyl-2-fluorenyl endgroups at their periphery were now prepared and studied. As for porphyrin analogues, it was found that an efficient energy transfer occurs from the peripheral fluorenyl units to the central phthalocyanine unit of these compounds, leading to intense red light emission and photosensitization of oxygen. While the linear optical properties of these new dendrimers are only slightly improved compared to those of their porphyrin-cored analogues for PDT and fluorescence imaging, their two-photon absorption (2PA) cross-sections are much more significantly boosted, evidencing the key role played by the central tetrapyrrolic unit in this respect. The impact of this structural change in relation with the impact resulting from the change in dendrimer generations are then discussed with the help of DFT and molecular dynamic calculations.

\section{Keywords}

Phthalocyanine $\bullet$ Fluorenyl $\bullet$ Two-Photon Absorption $\bullet$ Dendrimers $\bullet$ Oxygen Sensitization 


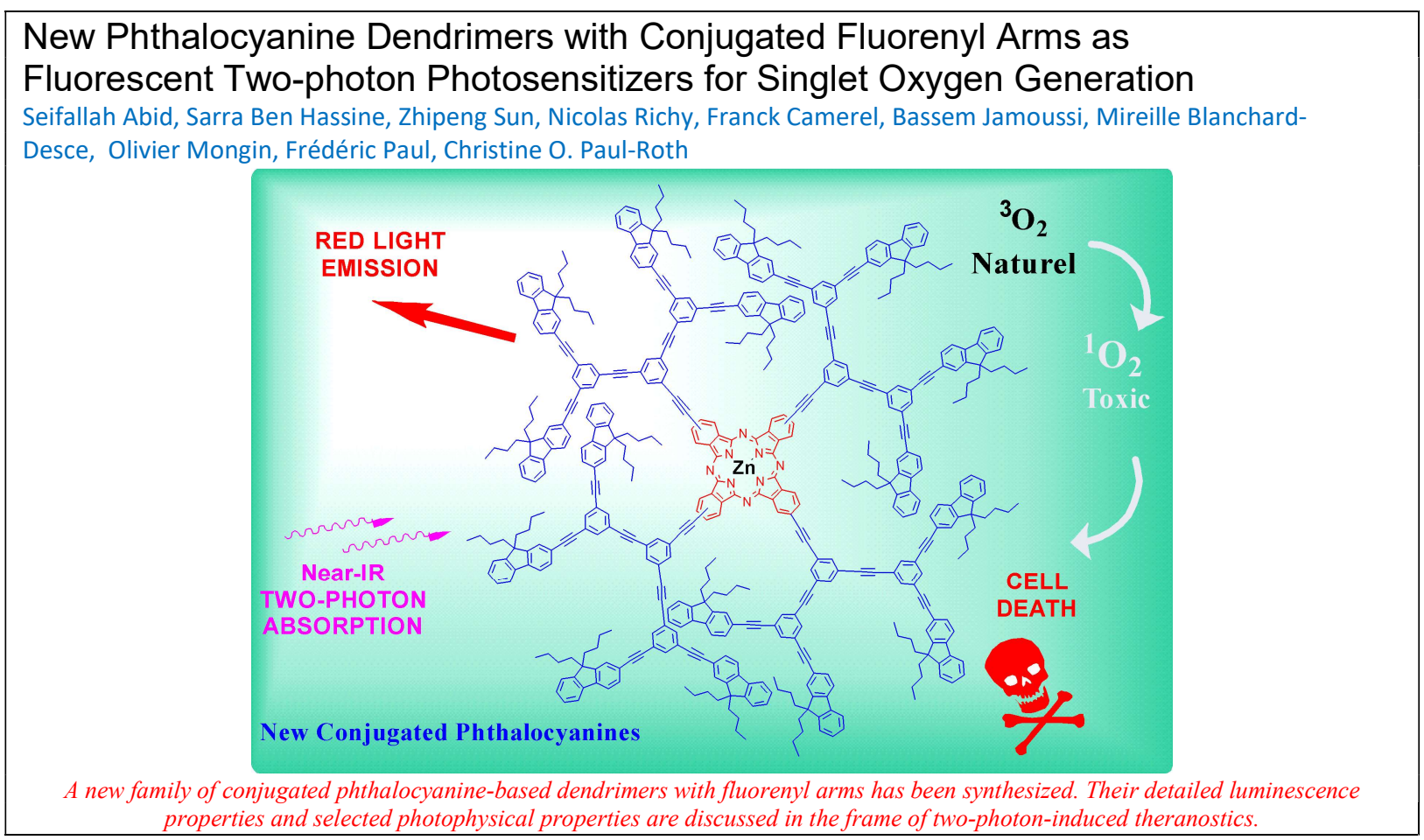




\section{- INTRODUCTION}

In the molecular world, following the seminal works of Vogtle, ${ }^{[1]}$ Tomalia, ${ }^{[2]}$ Newkome ${ }^{[3]}$ and others, ${ }^{[4]}$ dendrimers form nowadays a wonderful class of molecules presenting peripheral branches with a tree-like structure (dendrons) assembled around a central core. ${ }^{[5]}$ Such structures are usually obtained via the repetition of a similar sequence of reactions to increase the number of molecular units present within each dendron and therefore allowing to control several key properties of the dendrimer such as its size, its shape and the relative positions of various functional groups located at its periphery. ${ }^{[5]}$ In this respect, it was shown that dendrons can work as efficient "light-harvesting antenna" in selected porphyrin-based dendrimers presenting molecular architectures recalling those collecting natural light in various photosystems. ${ }^{[6]}$ After photoexcitation, the dendrons at the periphery of these systems were demonstrated to quantitatively transfer their energy to the central porphyrin core. ${ }^{[7 \mathrm{a}, 7 \mathrm{~b}, 7 \mathrm{c}-\mathrm{e}]}$ Their chemical structure proves therefore determining for this remarkable process to occur. For instance, with dendrons made of benzyl polyether chains, Fréchet demonstrated that the "antenna effect" was more efficient in branched architectures than in star-shaped architectures. ${ }^{[7 b]}$ Later on, fluorenyl units were identified as efficient aromatic chromophores in such systems, allowing to perform directly two-photon excitation of the peripheral dendrons ${ }^{[8]}$ or promoting 2PA via the first $2 \mathrm{PA}$-allowed excited states. ${ }^{[9]}$

With closely related dendrimers, we have previously shown than when progressing from the derivatives 1a-c ${ }^{[9 b, 10 a-c, 10 d]}$ to their more rigid carbon-rich analogues $\mathbf{2 a - c ^ { [ 1 1 ] }}$ (Scheme 1), the figures of merit for two-photon excited fluorescence and oxygen photosensitization were significantly improved. ${ }^{[11 \mathrm{~b}]}$ Thus, for nearly similar emission and oxygen photosensitization quantum yields, the two-photon absorption (2PA) cross-sections $\left(\sigma_{2}\right)$ of $\mathbf{2 b - c}$ dramatically increased compared to those of $\mathbf{1 b}-\mathbf{c} .^{[9 b, 11 b]}$ Then, we could recently show that after proper functionalization, $\mathbf{2 a - b}$ give rise to fluorescent photosensitizers adapted to one-photon photodynamic therapy (PDT), ${ }^{[12]}$ but also to more advanced developments, such as two-photon PDT (2P-PDT) ${ }^{[13]}$ or even theranostic ${ }^{[14]}$ uses. $^{[15]}$ More generally, this result suggested that replacing the polyether backbone of dendrimers such as 1a-c by a polyarylalkynyl backbone constitutes a way to significantly boost their biphotonic photosensitizing and emissive performances. If correct, this hypothesis provides a simple design criterium for improving the performances of related dendritic systems, especially when these are intended to be used as luminescent photosensitizers. 


\section{Previous scope}

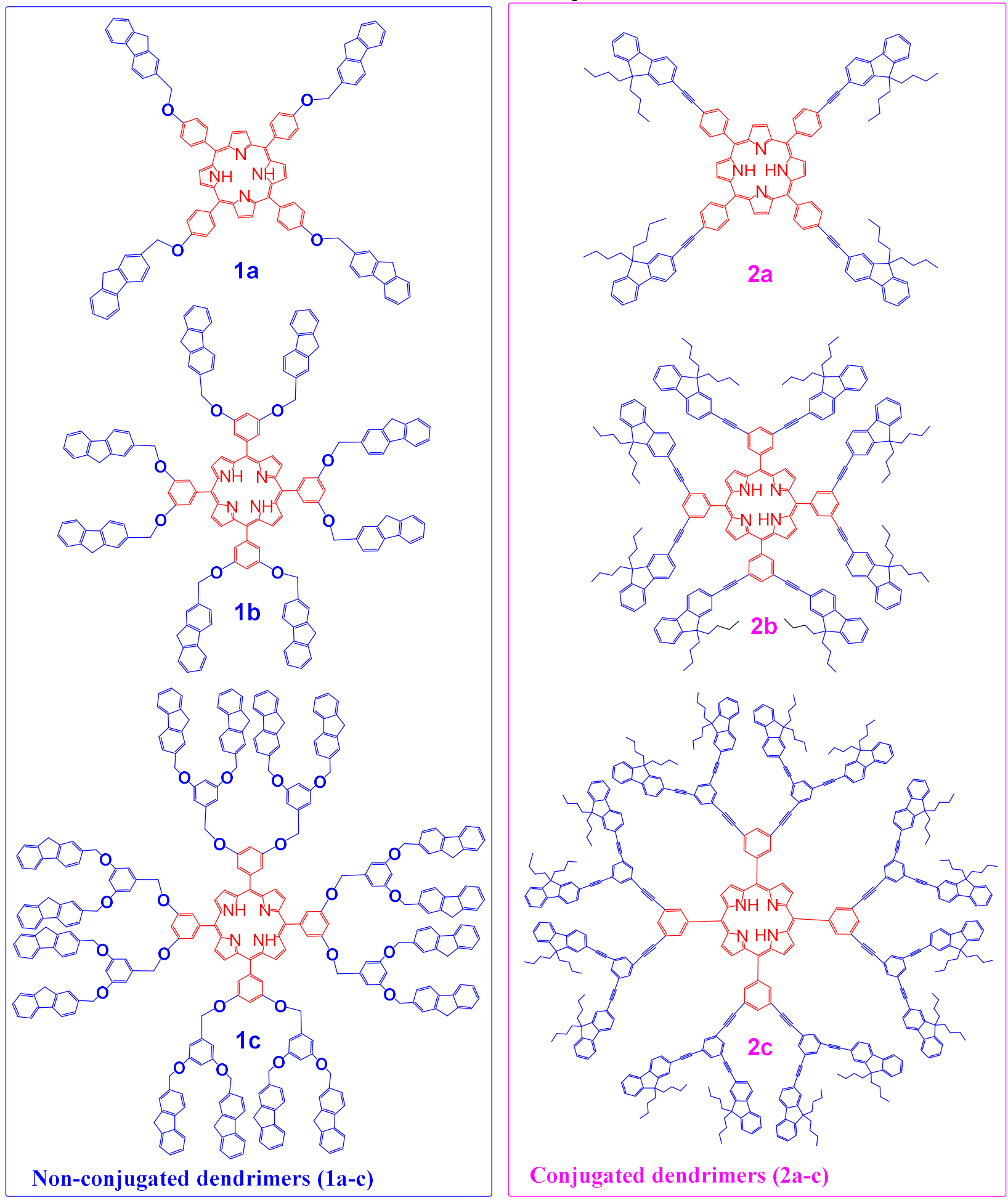

Scheme 1. Molecular structures of increasing generations of TPP-based dendrimers with non-conjugated (1a-c) or conjugated (2a-c) peripheral fluorenyl arms.

Motivated by further enhancing the efficiency of such systems and inspired by the phthalocyanine-cored dendrimers reported by Kimura ${ }^{[16]}$ and others, ${ }^{[17]}$ we recently wondered 
about the impact of replacing the central free-base meso-tetraphenylporphyrin ( $\left.\mathbf{H}_{2} \mathbf{T P P}\right)$ by zinc(II) phthalocyanine ( $\mathbf{Z n P c}$ ). Thus, we synthesized then compounds 3-5 and probed their photonic properties (Scheme 2). ${ }^{[18]}$ The idea behind this structural variation was to enhance the emission of these photosensitizers by using a more luminescent tetrapyrrolic central unit than H2TPP, but also able to activate oxygen with similar efficiency. This goal was perfectly reached with phthalocyanines 3-5 since all these compounds proved more fluorescent than porphyrins 1a-c (with luminescence quantum yields around 29-37\%) and even presented an improved twophoton photosensitizing ability for oxygen in THF. For these phthalocyanines, the comparison with the naked ZnPc complex also demonstrated the critical role of the peripheral fluorenylcontaining antenna on the relevant biphotonic properties. ${ }^{[18]}$

\section{Previous scope}

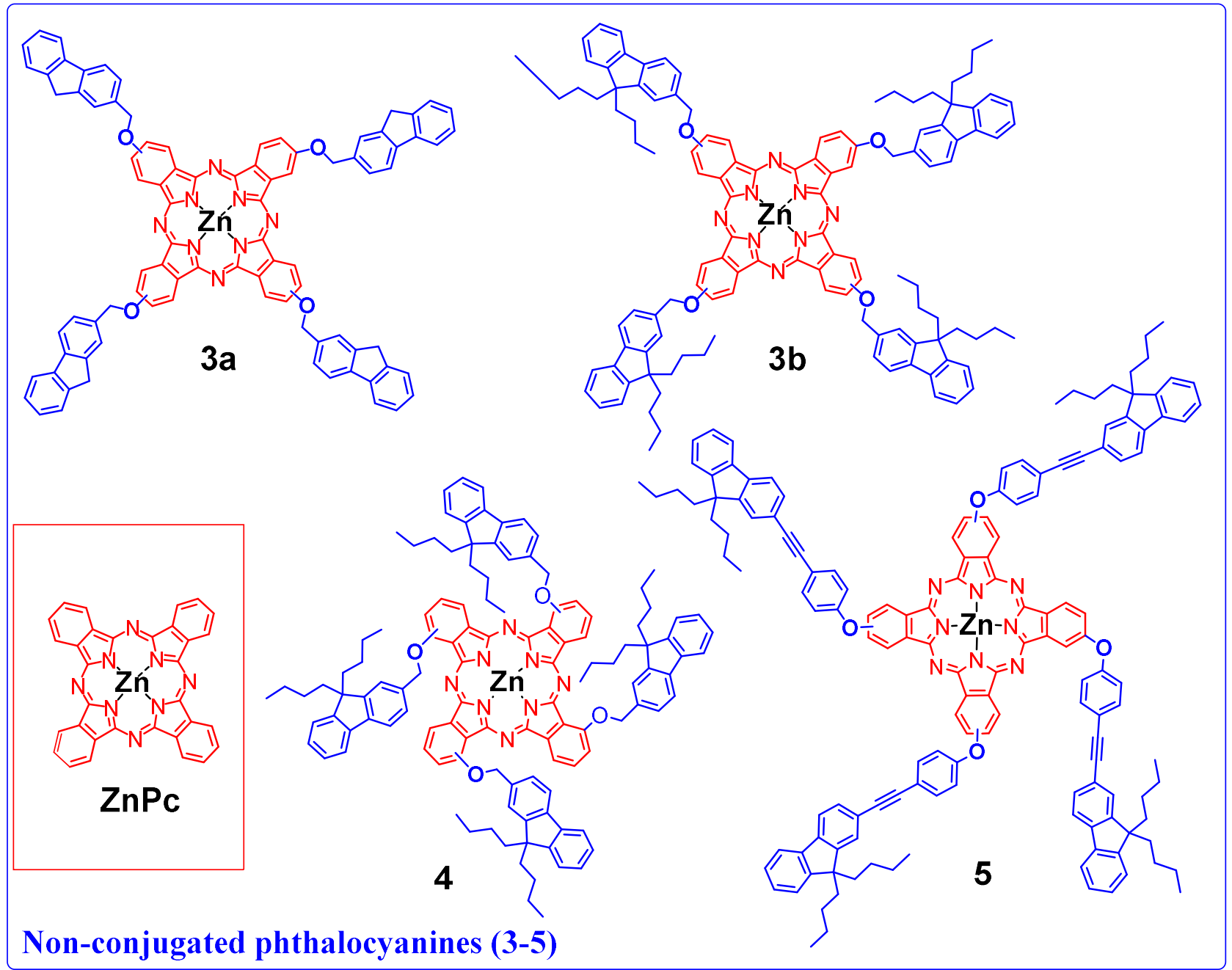

Scheme 2. Reference compound (ZnPc) and substituted phthalocyanines with non-conjugated alkoxy and phenoxy ether-based arms (3-5). 
Thus, these phthalocyanine-cored assemblies 3-5 exhibited larger intrinsic 2PA cross-sections which somewhat increased with the size of the $\pi$-manifold appended to the peripheral $(\beta)$ positions. Quite remarkably, the cross-section found for 5 featuring extended phenylalkynyl spacers was significantly above these of the larger porphyrin-based dendrimers such as $\mathbf{1 b}$-c or $\mathbf{2 b}$-c, resulting in better figures of merit for two-photon imaging or two-photon sensitization of oxygen in THF. Based on the previous observations made for porphyrin-based dendrimers (see above), it appeared likely to us that the photonic properties of these phthalocyanine-based systems might be further improved by moving to more conjugated carbon-rich backbones and also to dendrimers of higher generations.

\section{This work}

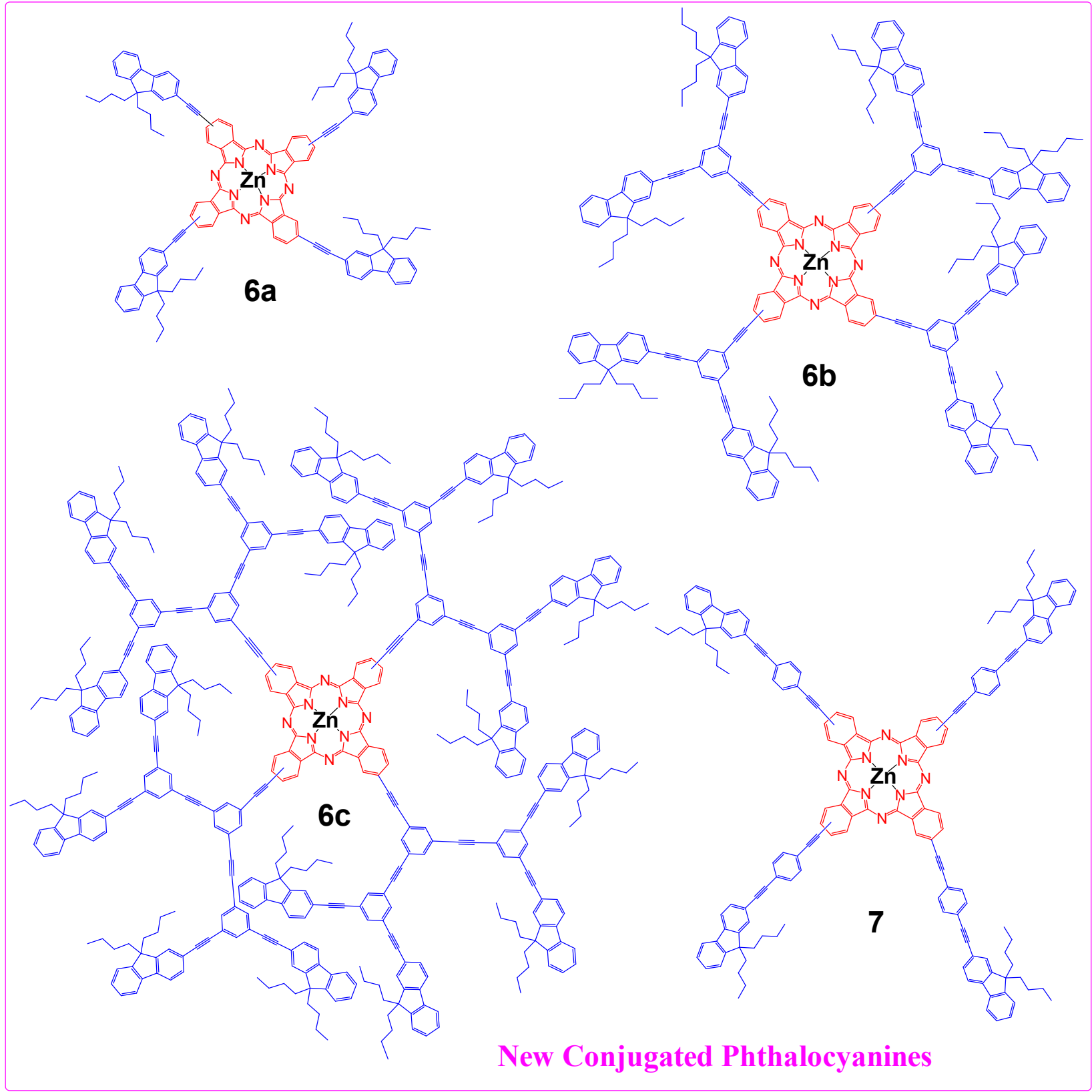

Scheme 3. Targeted phthalocyanines dendrimers 6a-c and star shaped 7 (the extended analogue of 6a). 
Accordingly, we have now decided to investigate the photonic properties of the set of new dendrimers 6a-c and 7 (Scheme 3), featuring polyarylethynyl dendrons similar to these present in previous porphyrins 2a-c (Scheme 1). We will therefore describe hereafter the isolation and characterization of these new dendrimers using an efficient and convergent synthetic approach. Subsequently, we will describe their linear and nonlinear optical (NLO) properties, as well as their oxygen photosensitizing capabilities, before briefly discussing their electronic structure and spatial organization with the help of DFT calculations. Notably, while several classes of phthalocyanines have already been used for imaging or PDT, ${ }^{[19]}$ to the best of our knowledge, such "carbon-rich" phthalocyanine-cored dendrimers have never been envisioned for such applications and their biphotonic properties are still unknown.

\section{RESULTS AND DISCUSSION}
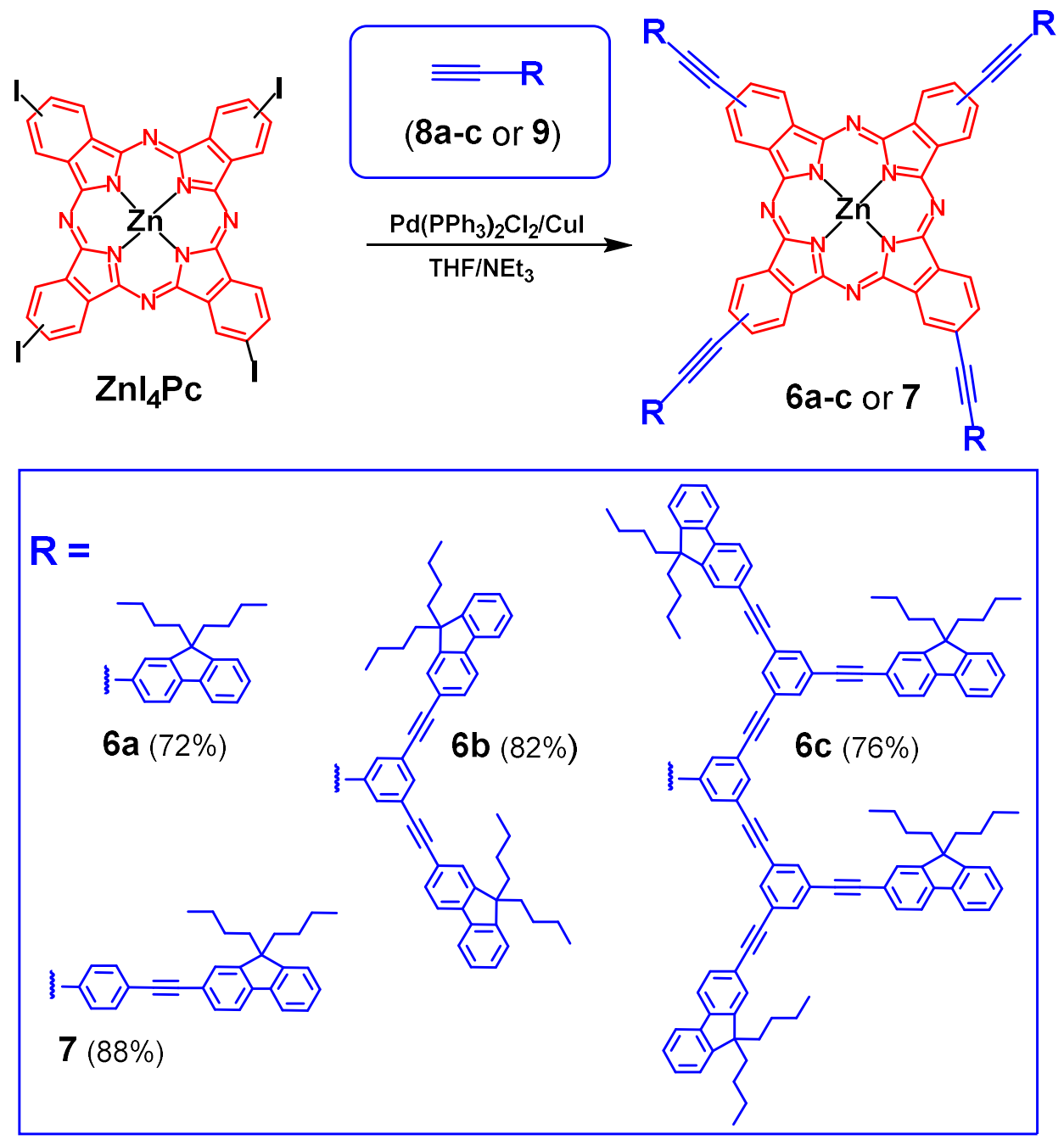

Scheme 4. Synthesis of new conjugated dendrimers 6a-c and Pc 7. 
We synthesized these new dendrimers 6a-c and phthalocyanine 7 (the extended analogue of 6a), by using a strategy similar to that previously used in our group to synthesize consecutive generations of tetrathienylporphyrin-cored dendrimers. ${ }^{[20]}$ It basically involves three steps; (i) synthesis of dendritic alkyne precursor, (ii) formation of the tetra-iodo macrocycle core and (iii) coupling of four alkyne-terminated dendrons (8a-c and 9) to the central core. Such a convergent synthetic approach was preferred over alternative ones because the targeted dendrimers proved sufficiently soluble to be easily purified by chromatography. The zinc(II) complex of tetraiodophthalocyanine (ZnI4Pc in Scheme 4), our starting platform described in 1998 by T. Torres ${ }^{[21]}$ was used as a common intermediate to access the four targeted molecules 6a-c and 7 , featuring up to sixteen fluorenyl groups. To do so, a fourfold Sonogashira coupling was carried out at ambient temperature in tetrahydrofuran-triethylamine mixtures. Whereas the alkynes 8ab and 9 were known compounds, ${ }^{[22]} \mathbf{8 c}$ was obtained in two steps by a Corey-Fuchs reaction from the known aldehyde precursor $\mathbf{1 0}^{[11 \mathrm{~b}]}$ with a total yield of $71 \%$ (Scheme 5 ).
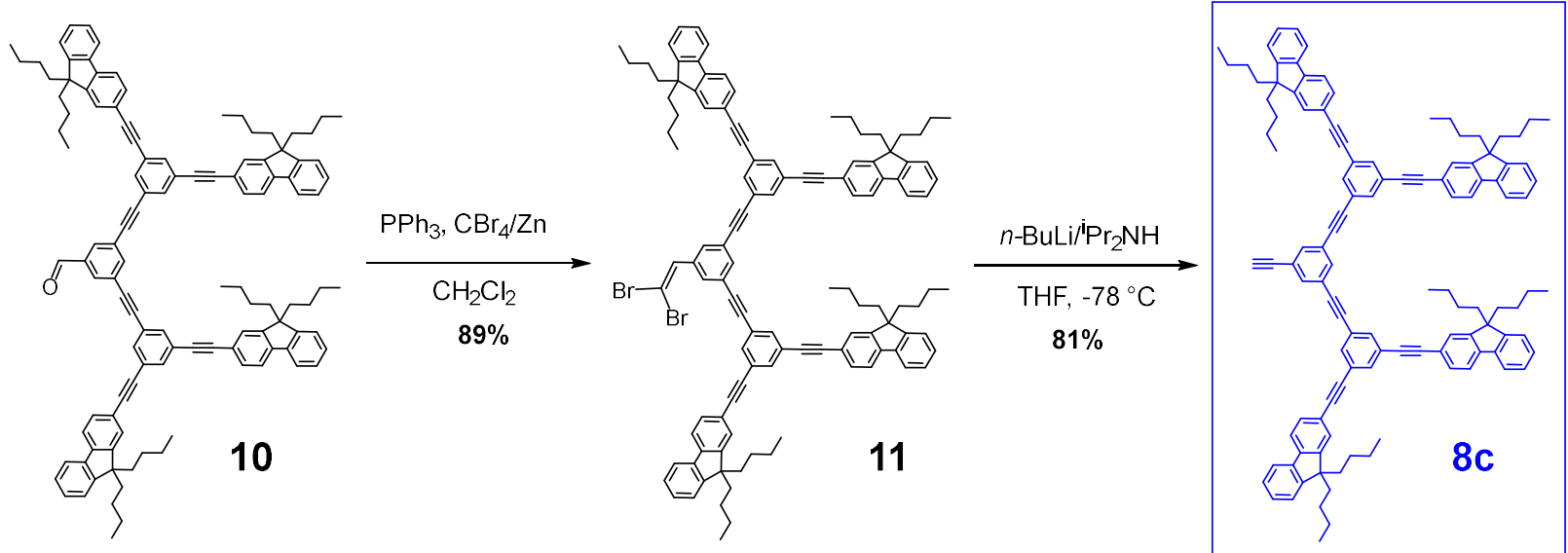

Scheme 5. Synthesis of new dendron precursor $\mathbf{8 c}$.

Compared to the more classical method consisting in cyclization of pre-functionalized phthalonitriles (the yield of which does generally not exceed $40 \%$ ), this convergent approach gave remarkably good yields (higher than $70 \%$ for each compound). For instance, when the synthesis of $\mathbf{6 a}$ was attempted by cyclizing 4-((9,9-dibutyl-9H-fluoren-2yl)ethynyl)phthalonitrile (12) in presence of zinc(II) acetate, the phthalocyanine 6a was only isolated with a total yield of $23 \%$ (Scheme 6). 


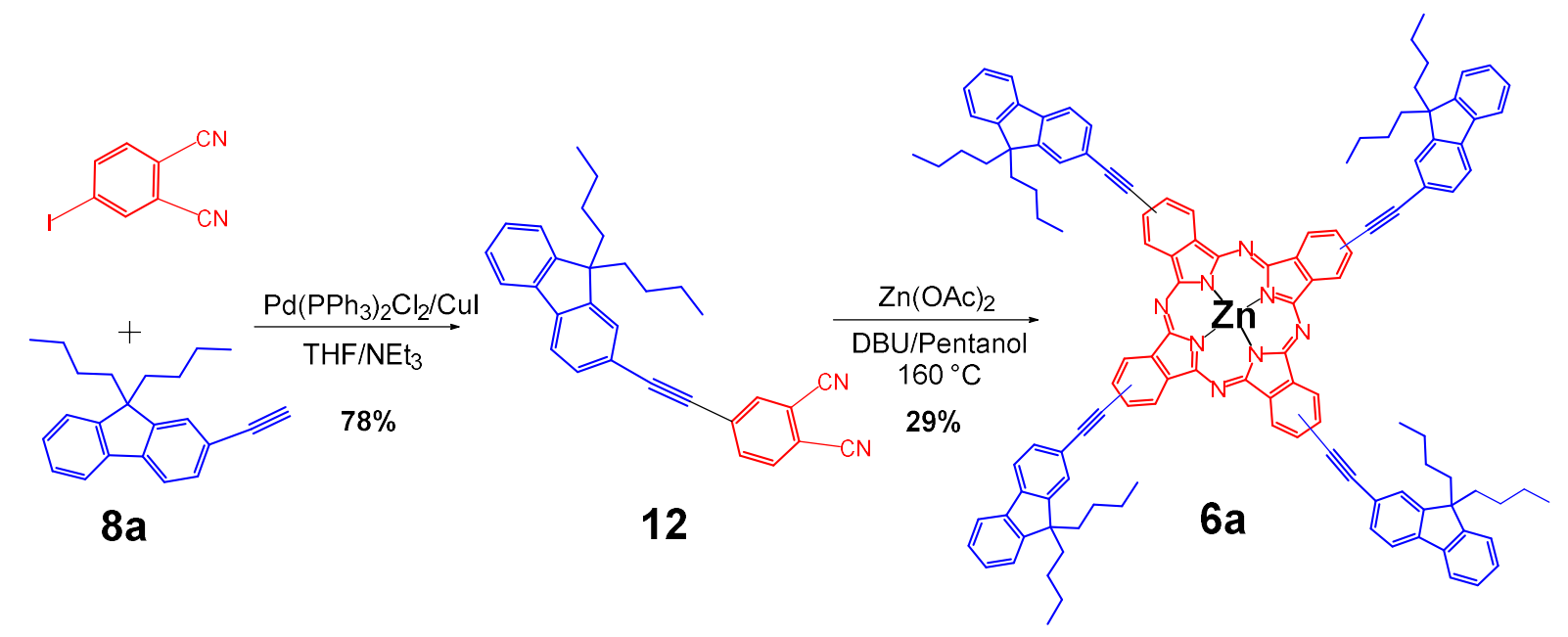

Scheme 6. Alternative synthesis of zinc complex $\mathbf{6 a}$.

Characterization of the Dendrimers. After chromatographic purification and recrystallization, all dendrimeric (6a-c) and star-shaped (7) compounds were characterized by usual techniques, including elemental analysis. Thanks to the $n$-butyl chains on the peripheral fluorene groups, they all exhibited sufficient solubilities in THF for NMR characterization, in which solvent they appeared to be essentially non-aggregated, at least below concentrations of $10 \mathrm{mM}$ (Figure 1a). ${ }^{123]}$ This is remarkable, since the low solubility of phthalocyanines, which usually results from their tendency to aggregate in solution, ${ }^{[22,24]}$ is often an obstacle to their NMR characterization and practical use. Moreover, based on the previous investigation with phthalocyanines 3a-b (Scheme 2), ${ }^{[18]}$ the introduction of the various $n$-butyl chains should have almost no effect on their electronic structure nor on their optical properties, apart perhaps slightly decreasing their fluorescence quantum yields.

As expected, ${ }^{1} \mathrm{H}$ NMR confirms that 6a-c and 7 are isolated as mixtures of positional regioisomers (Figure 1b), the phthalocyanine protons being observed as large multiplets in line with the presence of overlapping sets of closely lying signals belonging to the various regioisomers. Thus, their ${ }^{1} \mathrm{H}$ NMR spectra present diagnostic signals in three distinct spectral ranges (Figure 1a); (i) between 8.1 and $9.6 \mathrm{ppm}$ for the twelve phthalocyanine protons; (ii) between 7.2 and 8 ppm for the aromatic protons of the fluorenyl and phenyl groups and (iii) between 0.5 and $2.4 \mathrm{ppm}$ for the aliphatic protons of the $n$-butyl chains. In line with the increasing number of peripheral fluorenyl, the intensity of the two latter sets of signals increases

\footnotetext{
${ }^{1}$ Notably, all these compounds were amenable to partial ${ }^{13} \mathrm{C}$ NMR characterization in THF-d ${ }^{8}$.
} 
relative to that of the low field phthalocyanine signals (i) along the series 6a-c.

(a)

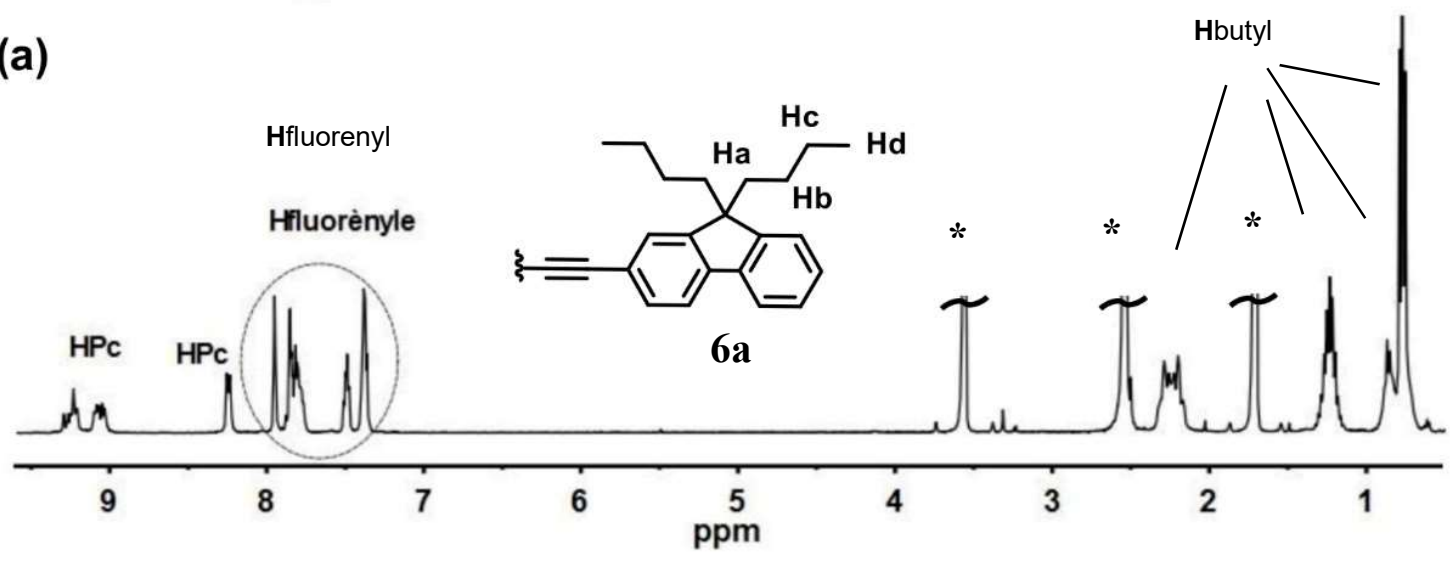

(b)
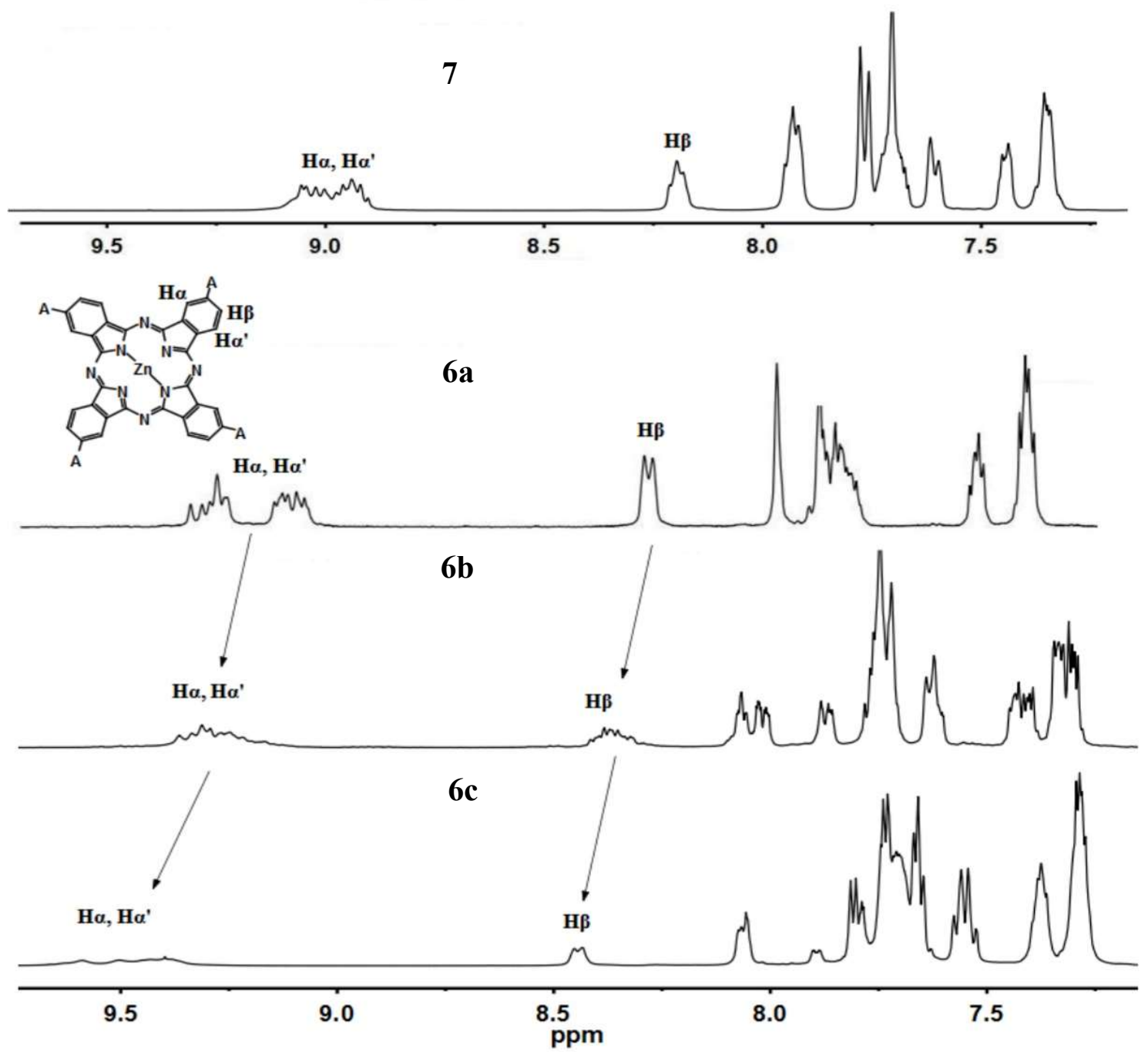

Figure 1. a) Complete ${ }^{1} \mathrm{H}$ NMR spectra of phthalocyanine 6a in THF- $\mathrm{d}_{8}$ (solvent and water peaks labelled by asterix); b) Low-field section of the ${ }^{1} \mathrm{H}$ NMR spectra of phthalocyanine derivatives $\mathbf{7}$ and $\mathbf{6 a - c}$ in THF- $\mathrm{d}_{8}$. 
A slight downfield shift of the signals corresponding to the twelve protons of the central phthalocyanine core can also be noticed when progressing from 6a to 6c (Figure 1b). By comparison, an upfield shift of these protons is stated when proceeding from $\mathbf{6 a}$ to 7 . These shifts, which are not observed for the other aromatic protons, likely reflect a change in the magnetic shielding of the central tetrapyrrolic unit. The latter could result from ring current effects originating from the specific conformations adopted in solution by the peripheral dendrons in the higher generations dendrimers (see DFT section).

One- and two-photon absorption (1PA and 2PA) and emission properties, as well as oxygen photosensitization properties were next determined for 6a-c and 7 in THF solution (Table 1), using the zinc phthalocyanine ZnPc as a reference. The UV/Vis absorption spectra of all these new phthalocyanines and of the starting zinc complex $\mathbf{Z n I} \mathbf{I}_{4} \mathbf{P c}$ were recorded between 250 and $800 \mathrm{~nm}$ (Figure 2). Without surprise, the spectrum of the latter compound is almost similar to that of the reference compound ZnPc. The new compounds $\mathbf{6 a - b}$ and $\mathbf{7}$ also feature the characteristic absorption bands of phthalocyanines already seen on the spectra of $\mathbf{Z n P c}$ and $\mathbf{Z n I}_{4} \mathbf{P c},{ }^{[25]}$ which are: (i) the Q-band, corresponding to the main absorption band, detected in the visible range between 650 and $750 \mathrm{~nm}$, and resulting from $\pi$ - $\pi^{*}$ transitions from the HOMO to the LUMO and LUMO+1 with a vibronic sub-band around $630 \mathrm{~nm} ;{ }^{2[17 \mathrm{~g}, 22,26]}$ (ii) the B- (or Soret) band, corresponding to a wide band between 300 and $500 \mathrm{~nm}$ and resulting from $\pi-\pi^{*}$ transitions from deeper lying MOs to the LUMO and LUMO+1, and, finally, (iii) the $\mathrm{N}$ band, a less intense and narrower band centered around 280-300 $\mathrm{nm}$. The latter still corresponds to a set of more energetic $\pi-\pi^{*}$ transitions within the phthalocyanine core. These last two absorptions are not plainly detected for $\mathbf{6 a - b}$ and $\mathbf{7}$ because they are overlapped by another intense absorption band, absent in $\mathbf{Z n P c}$ and $\mathbf{Z n I}_{\mathbf{4}} \mathbf{P c}$, which grows in intensity and shifts toward the blue when progressing from 7 to $\mathbf{6 a}$ and then to $\mathbf{6 b}-\mathbf{c}$ (Figure 2) i.e. roughly with the increasing size of the conjugated $\pi$-manifold within the arms. This broad band, which becomes more intense than the Q-band for the two largest dendrimers (6b-c), can be confidently attributed to dendron-centered $\pi-\pi^{*}$ transitions. When this absorption is compared to these previously observed around $300 \mathrm{~nm}$ with phthalocyanines presenting non-conjugated dendrons, such as 3b-5 (Scheme 2), ${ }^{[18]}$ a red shift is stated for it in $\mathbf{6 a - c}$ and 7 . This shift reflects at least in part the better $\pi$-conjugation existing between the peripheral arms and core in these

\footnotetext{
${ }^{2}$ In line with the ${ }^{1} \mathrm{H}$ NMR data, the shape of the Q-band (with no shoulder), which remains constant throughout the whole series of new compounds, is suggestive of absence of aggregates in solution, at the concentrations used $\left(10^{-4}-10^{-7} \mathrm{M}\right){ }^{[39,51,56]}$
} 
compounds. In a similar way, a bathochromic shift is experienced by the main Q-band following roughly the same order. Thus, this maximum shifts towards the red, from 674 to $697 \mathrm{~nm}$, along the order: $\mathbf{Z n P c}<\mathbf{Z n I} 4 \mathbf{P c}<\mathbf{6 b} \approx \mathbf{6 c}<\mathbf{6 a} \approx \mathbf{7}$. The Q-band of 6a-c and 7 grows in intensity following roughly the same order, the star-shaped phthalocyanine 6a presenting the most shifted and strongest Q-band. Again, this shift reflects the importance of the $\pi$-manifold present on the peripheral dendrons, conjugating with the central core, in line with the existence of an effective mesomeric interaction between these parts in 6a-c and 7.

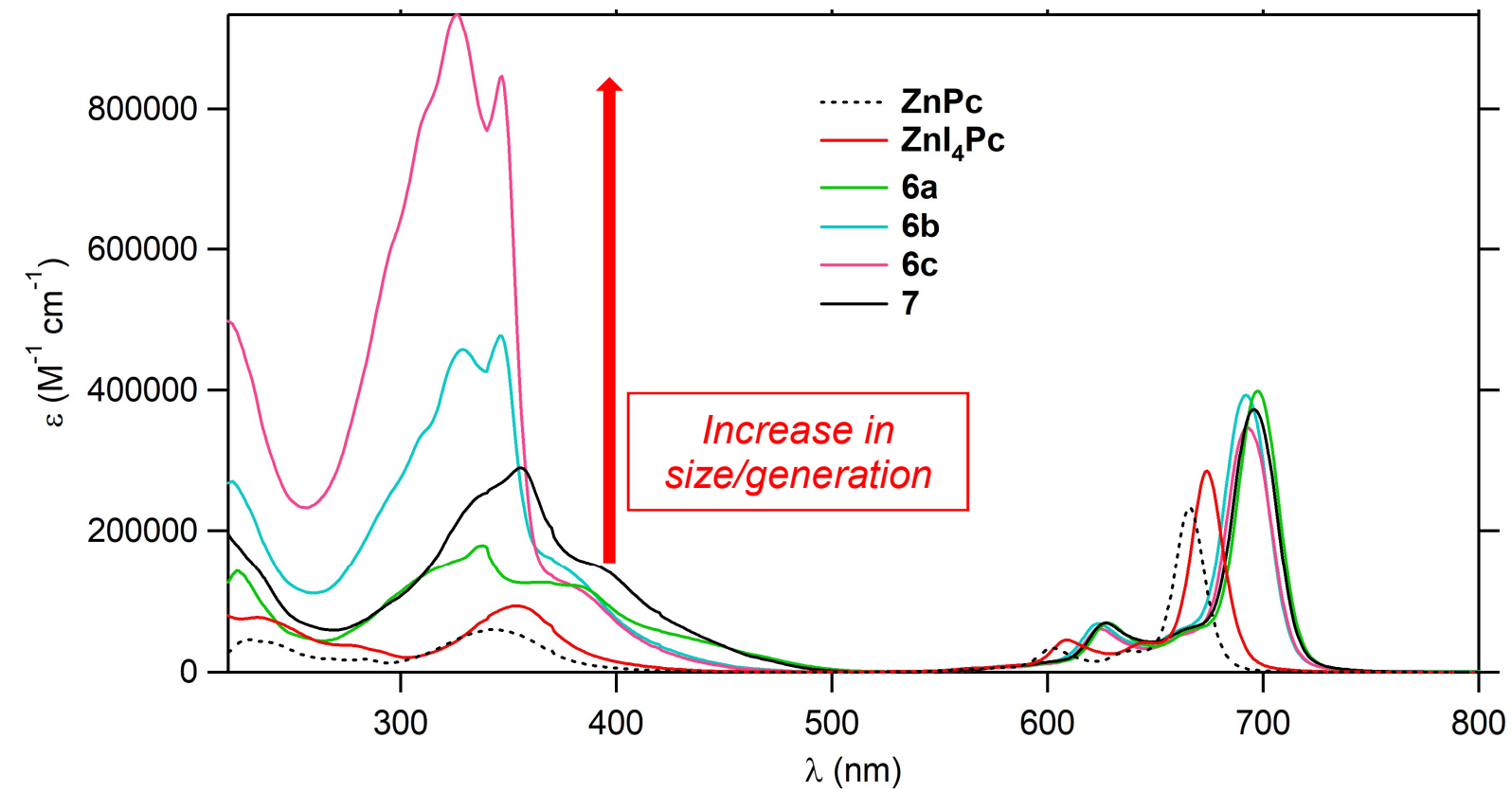

Figure 2. The UV-visible absorption spectra of the new series 6a-c and 7, the $\mathbf{Z n I} \mathbf{I}_{4} \mathbf{P c}$ precursor and $\mathbf{Z n P c}$ used as a reference in THF.

Figure 3 shows the emission spectra of dendrimers 6a-c and 7 in THF, as well as that for their synthetic precursor $\left(\mathbf{Z n I}_{\mathbf{4}} \mathbf{P c}\right)$ and also that of $\mathbf{Z n P c}$ used as reference. After excitation at $606 \mathrm{~nm}$ i.e. in the Q vibronic sub-band, a strong red emission between 650 and $800 \mathrm{~nm}$, characteristic of the phthalocyanine core, is observed. As for one-photon absorption, these dendrimers present a red-shifted emission relative to that of $\mathbf{Z n I}_{4} \mathbf{P c}$ and $\mathbf{Z n P c}$. Without surprise, the wavelength ordering between the new compounds follows that previously observed in absorption for the Q-band $\left(\mathbf{Z n P c}<\mathbf{Z n I}_{4} \mathbf{P c}<\mathbf{6 b} \approx \mathbf{6 c}<\mathbf{7} \approx \mathbf{6 a}\right)$. 

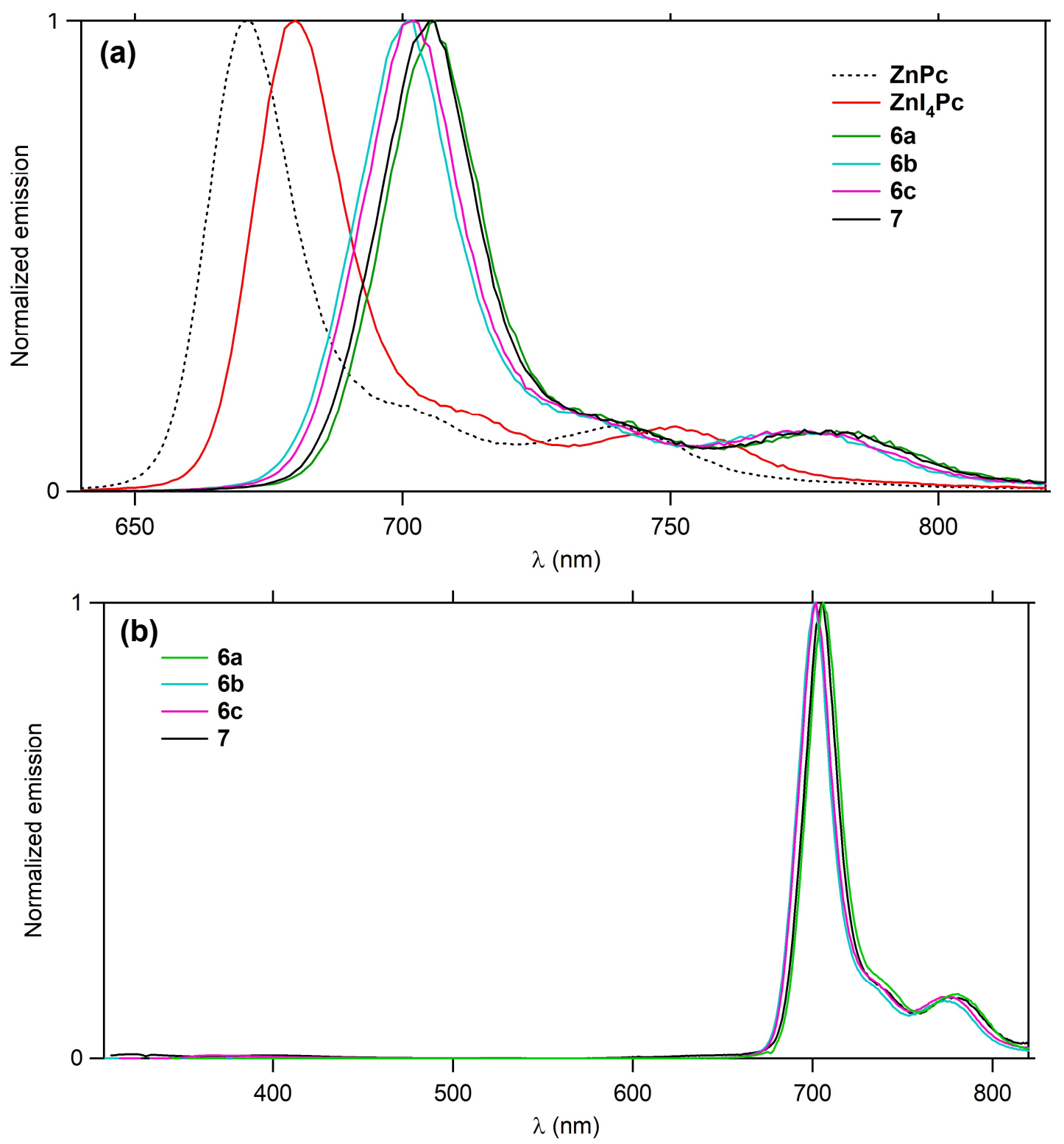

Figure 3. a) Normalized emission spectra of dendrimers 6a-c, 7, ZnI4Pc and ZnPc after excitation at $606 \mathrm{~nm}$ in THF; b) Normalized emission spectra of 6a-c and 7 under similar conditions after excitation at $300 \mathrm{~nm}$.

As previously done with phthalocyanines $3-5,{ }^{[18]}$ the fluorescence quantum yields $\left(\Phi_{\mathrm{F}}\right)$ were determined by comparison with $\mathbf{Z n P c}$ in a toluene/pyridine mixture (99:1). Compared to this standard $\mathbf{Z n P c}\left(\Phi_{\mathrm{F}}=26 \%\right.$ ), all quantum yields found for the new compounds are higher than this value, excepted for $\mathbf{Z n I}_{4} \mathbf{P c}\left(\Phi_{\mathrm{F}}=11 \%\right)$. Actually, among the dendrimer series 6a-c, there is a regular increase in their fluorescence quantum yield with increasing generations $(29 \%$ $<32 \%<36 \%$ ), suggesting that the increase of the $\pi$-manifold on the peripheral dendrons favors the non-radiative deactivation of the phthalocyanine core. When $\mathbf{6 a}$ and $\mathbf{7}$ are compared, a marginally better quantum yield $(30 \%)$ is found for 7 , possibly in line with the previous observation. When compared to the fluorescence quantum yield of zinc complexes 3-5 
previously investigated $(29-37 \%),{ }^{[18]}$ these quantum yields span a nearly similar range of values, but only the yield found for largest compound $\mathbf{6 c}$ compares to that previously obtained for 5, which was the most luminescent compound among 3-5 series.

Then, to probe the efficiency of the energy transfer process (EnT) between the peripheral dendrons as energy donors and the central phthalocyanine as energy acceptor, we have excited the dendrimers in their specific $\pi-\pi^{*}$ band, at about $300 \mathrm{~nm}$ (Figure 3b). By doing so, we observed no residual emission of the dendrons in the $350-450 \mathrm{~nm}$ range, ${ }^{[10 \mathrm{~b}, 11 \mathrm{~b}, 27]}$ but only the characteristic emission band from the phthalocyanine core (between 650 and $800 \mathrm{~nm}$ ), with similar fluorescence quantum yields than when the compounds are excited in their Q-band. This reveals that for all these derivatives, the energy absorbed by the dendrons is almost quantitatively transferred to the central macrocycle, pointing to an optimal process $\left(\Phi_{\mathrm{EnT}} \sim\right.$ $100 \%$ ), regardless of the generation considered.

For one-photon (1PA) fluorescence imaging purposes, one-photon brightness $\left(\varepsilon_{\mathrm{ex}} . \Phi_{\mathrm{F}}\right)$ must be considered. ${ }^{[28]}$ This figure of merit depends on the fluorescence quantum yield and on the absorption coefficient at the excitation wavelength $\left(\varepsilon_{\mathrm{ex}}\right)$. Thus, when excitation is performed at the maximum of the Q-band, comparable or better one-photon brightnesses are obtained for 6a-c than for $\mathbf{5}$. As a result, all these compounds will perform better than the phthalocyanines 3b-5 previously studied. Notably, thanks to the efficient energy transfer previously discussed, significantly improved 1PA brightnesses (around 153000 and $336000 \mathrm{M}^{-1} \mathrm{~cm}^{-1}$, respectively) can be obtained for $\mathbf{6 b}$ and $\mathbf{6 c}$ if the excitation is performed at the wavelength corresponding to the dendron-specific $\pi-\pi^{*}$ absorption band (around $320 \mathrm{~nm}$ ). Therefore, imaging might be more efficiently performed by exploiting the light harvesting capability of these dendrimers, by using a more energetic (blue shifted) excitation light beam, although the gain will be achieved at the expense of the penetration depth of the exciting light beam and might induce potentially larger background fluorescence due to intrinsic fluorophores. 
Table 1. Photophysical properties of the zinc phthalocyanine complexes and of the reference ZnPc in THF at $293 \mathrm{~K}$. Related data are also given for selected phthalocyanines in THF.

\begin{tabular}{|c|c|c|c|c|c|c|c|c|c|c|c|c|}
\hline Cpnd & $\lambda_{\mathrm{abs}}{ }^{\text {Dend }}$ & $\lambda_{\mathrm{abs}}{ }^{\mathrm{B}}$ & $\lambda_{\text {abs }}{ }^{Q}$ & $\varepsilon_{\max }^{\text {Den }}$ & $\varepsilon_{\max } \mathbf{Q}$ & $\lambda_{\mathrm{em}}$ & $\Phi_{\mathrm{F}}^{\mathrm{a}}$ & $\varepsilon_{\max }{ }^{\text {Den }} \cdot \Phi_{\mathbf{F}}{ }^{b}$ & $\varepsilon \max ^{Q} \cdot \Phi_{F}^{b}$ & $\Phi_{\Delta}{ }^{\mathrm{c}}$ & ${ }^{\varepsilon \max }{ }^{\text {Den }} \cdot \Phi_{\Delta}{ }^{\mathrm{b}}$ & $\varepsilon \max ^{\mathrm{Q}} \cdot \Phi_{\Delta}^{\mathrm{d}}$ \\
\hline & \multicolumn{3}{|c|}{$(\mathbf{n m})$} & \multicolumn{2}{|c|}{$\left(10^{3} \mathrm{M}^{-1} \cdot \mathrm{cm}^{-1}\right)$} & \multicolumn{2}{|l|}{$(\mathbf{n m})$} & \multicolumn{2}{|c|}{$\left(10^{3} \mathrm{M}^{-1} \cdot \mathrm{cm}^{-1}\right)$} & & \multicolumn{2}{|c|}{$\left(10^{3} \mathrm{M}^{-1} \cdot \mathrm{cm}^{-1}\right)$} \\
\hline $\mathrm{ZnPc}$ & - & 343 & 602,666 & / & 234.0 & 671,741 & $0.26^{\mathrm{c}}$ & / & 60.8 & 0.61 & / & 142.7 \\
\hline $\mathrm{ZnI}_{4} \mathrm{Pc}$ & - & 343 & 609,674 & / & 285.0 & 680,751 & 0.11 & I & 31.3 & - & l & - \\
\hline $3 b$ & 276,306 & 351 & 611,677 & 107 & 215.0 & 688,758 & 0.33 & 35.3 & 71.0 & 0.57 & 61.0 & 122.6 \\
\hline 4 & 270,307 & 353 & 628,698 & 100 & 270.0 & 706,779 & 0.23 & 23.0 & 62.1 & 0.60 & 60.0 & 162.0 \\
\hline 5 & 327,344 & $\mathrm{f}$ & 609,675 & 253 & 291.0 & 683,753 & 0.37 & 93.6 & 107.7 & 0.54 & 136.6 & 157.1 \\
\hline $6 a$ & 338 & $\mathrm{f}$ & 628,697 & 179 & 398.0 & 706,781 & 0.29 & 51.9 & 115.4 & 0.60 & 107.4 & 238.8 \\
\hline $6 b$ & 329,346 & f & 624,692 & 478 & 393.0 & 701,774 & 0.32 & 153.0 & 125.8 & 0.51 & 243.8 & 200.4 \\
\hline $6 c$ & 226,347 & f & 624,693 & 933 & 347.0 & 702,774 & 0.36 & 335.9 & 124.9 & 0.53 & 494.5 & 183.9 \\
\hline 7 & 356 & f & 627,696 & 289 & 373.0 & 705,778 & 0.30 & 86.7 & 111.9 & - & - & - \\
\hline
\end{tabular}

${ }^{a}$ Fluorescence quantum yield determined relative to (Py)ZnPc in a toluene/pyridine (99:1) mixture after excitation at 606 nm. ${ }^{[29]}{ }^{b}$ One-photon brightness. ${ }^{[28]}{ }^{c}$ Singlet oxygen formation in a toluene/pyridine (99:1) mixture relative to $\mathbf{Z n P c}\left(\Phi_{\Delta}=0.61\right)$ in the same mixture. ${ }^{[30]}{ }^{d}$ Figure of merit for $\Phi_{\Delta}$ equivalent to one-photon brightness. ${ }^{e}$ Lit. $\Phi_{\mathrm{F}}=$ 0.23 ( \pm 0.03$)$ determined in THF by the comparative method using chlorophyll in diethylether as a standard. ${ }^{[26]}{ }^{\mathrm{f}}$ Overlapped by the dendron absorption. 
To evaluate the oxygen photosensitizing properties of 6a-c, the singlet oxygen formation yields were determined by measuring the luminescence of the singlet oxygen photogenerated in a toluene/pyridine (99:1) mixture. The values obtained for these compounds show a first decrease upon progressing from generation $\mathrm{G}_{0}$ to $\mathrm{G}_{1}(60 \%$ for $\mathbf{6 a}$ as. $51 \%$ for $\mathbf{6} \mathbf{b})$ and then a slight increase from generation $\mathrm{G}_{1}$ to $\mathrm{G}_{2}(51 \%$ for $\mathbf{6 b} v s .53 \%$ for $\mathbf{6 c})$. These values are similar or slightly lower than that of the ZnPc reference compound (61\%). ${ }^{[30]}$ This can be explained considering that the increase in the fluorescence quantum yields stated for these dendrimers is obtained at the expense of the singlet oxygen production, as already stated for 35. ${ }^{[18]}$ Actually, the yield presently determined for $\mathbf{6 c}$ is nearly similar to that previously found for $\mathbf{5}$. Thus, when compared to phthalocyanines $\mathbf{3 b}-\mathbf{5}$, these results, along with the larger $\varepsilon_{\mathrm{ex}}{ }^{\mathrm{Q}} \cdot \Phi_{\mathrm{F}}$ products for 6a-c, make them comparably better one-photon photosensitizers for singlet oxygen generation than 5 when excitation is performed at the wavelength of the Q-bands (i.e. at 690$700 \mathrm{~nm}$ for 6a-c). Likewise, to what was previously stated for fluorescence imaging, using the light-harvesting capability of the peripheral dendrons, the performance of the higher generation dendrimers $\mathbf{6 b}$ and $\mathbf{6 c}$ for oxygen sensitization might be further improved by selecting an excitation corresponding to the dendrimer absorption band (320-350 nm). Again, the increase in photosensitizing efficiency will be achieved at the expense of some tissular penetration depth of the exciting light beam.

The two-photon absorption (2PA) cross-sections of 6a-c dendrimers were next determined by two-photon excitation fluorescence (TPEF) and compared to that of $\mathbf{Z n P c}$ also used here as a reference compound (Table 2). A totally quadratic dependence of the fluorescence intensity as a function of the laser excitation power was observed for each compound at all the wavelengths (Figures S15 and S16; ESI), indicating that the cross-sections $\left(\sigma_{2}\right)$ so determined are solely due to two-photon absorption. However, past a limit (typically below $840 \mathrm{~nm}$ ), which certainly corresponds to the upsurge of residual one-photon absorption, ${ }^{[31]}$ the quadratic dependence of emission intensity on the laser power is lost. Thus, below this wavelength no intrinsic 2PA cross-sections can be determined anymore (Figure 4). In such a case, one of the very specific advantages of 2PA for bio-oriented applications is lost; namely ultra-dense focalization of the excitation volume. ${ }^{[32]}$ As found previously for the phthalocyanines $\mathbf{3 - 5},{ }^{[18]}$ the cross-sections of all these compounds increase when the wavelength of the incoming laser beam decreases (i.e. when the incoming light becomes more energetic). The best $\sigma_{2}$ values are therefore obtained at the above-mentioned limit ( $840 \mathrm{~nm}$ ) for ZnI 4 Pc and 6a-c and 7 ( $\sigma_{2}{ }^{\text {lim; }}$ Table 2$)$. Among the new derivatives, significantly larger $\sigma_{2}$ values are found at this wavelength for the higher generation dendrimers $6 \mathbf{b}\left(\mathrm{G}_{1}\right)$ and $\mathbf{6} \mathbf{c}\left(\mathrm{G}_{2}\right)$.

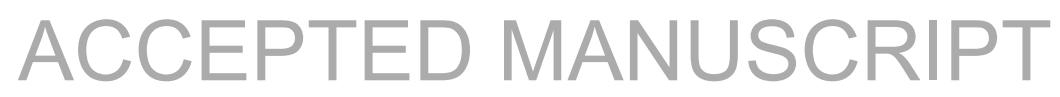


At this limit, 6a $\left(\mathrm{G}_{0}\right)$ absorbs significantly better than $\mathbf{Z n P c}$, while $\mathbf{Z n I} \mathbf{4} \mathbf{P c}$ is the least 2PAactive compound of this series. Thus, both $\mathbf{6 b}$ and $\mathbf{6 c}$ have $\sigma_{2}$ maxima of 1640 and $1880 \mathrm{GM}$, respectively, against $1010 \mathrm{GM}$ for $\mathbf{6 a}$ and $90 \mathrm{GM}$ for ZnI4Pc (Figure 4). At higher wavelengths, a maximum can then be detected as a peak or as a shoulder (around $900 \mathrm{~nm}$ ) on the 2PA plots recorded for 6a-c and $7\left(\sigma_{2}{ }^{\max }\right)$. While the intensities of these 2PA maxima appear slightly less sensitive to the dendrimer size than $\sigma_{2}{ }^{\lim }$ values, they present a similar size-dependence. In addition, a second 2PA maximum, around $960 \mathrm{~nm}(130 \mathrm{GM})$, can also be detected for small $6 \mathbf{6}$. As previously discussed, ${ }^{[18]}$ these different $2 \mathrm{PA}$ maxima can be attributed to population of various one-photon forbidden $g$ - $g$ excited states located at energies between these of the B-band and the Q-band, the nature of which being impossible to infer from the 1PA spectra (Figures S17 and S18; ESI). ${ }^{[3]}$ While, the exact nature of these states (to which the various dendrons might contribute) is certainly determining for establishing the 2PA cross-sections, the bathochromic shift experienced by the first Q-state in 6a-c and 7 possibly contributes to promote 2PA via a resonance enhancement mechanism, ${ }^{[33 \mathrm{a}]}$ especially when its energy comes closer to that of the exciting beam, as was previously demonstrated for phthalocyanines functionalized by conjugated carbon-rich substituents at one of their $\beta$-positions. ${ }^{3[32]}[8,34]$

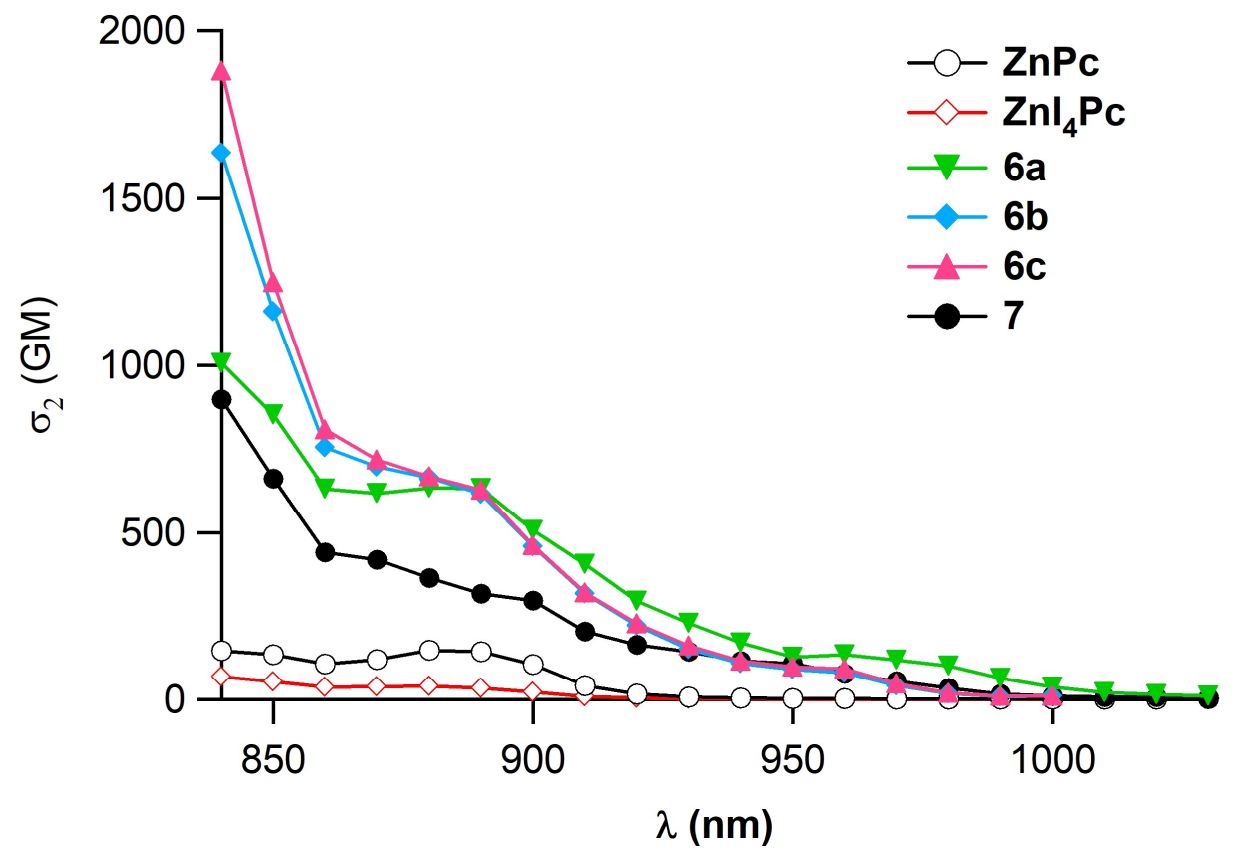

Figure 4. Two-Photon Absorption Spectra for phthalocyanine-Cored Dendrimers 6a-c, 7, ZnI $\mathbf{4} \mathbf{P c}$ and $\mathbf{Z n P c}$.

\footnotetext{
${ }^{3}$ Contrary to was done with related compounds for which the absorption of the peripheral substituents was used to boost the 2PA cross-section of the central tetrapyrrolic unit via a biphotonic light-harvesting process, $[13,14,16]$ the dendron-based state detected in the range 320-360 nm on 1PA spectra of 6a-c and 7 cannot be used for performing two-photon excitation of these nonlinear chromophores, since the required wavelengths $(640-720 \mathrm{~nm})$ would be below the limit $(840 \mathrm{~nm})$ where processes other than pure 2PA come in play.
} 
Table 2. Two-photon absorption of phthalocyanine $\mathrm{Zn}(\mathrm{II})$ complexes and related tetrapyrrolic macrocycles in $\mathrm{THF}$ at $20^{\circ} \mathrm{C}$

\begin{tabular}{|c|c|c|c|c|c|c|c|c|c|c|c|c|}
\hline Cpnd & $\begin{array}{c}\lambda_{\mathrm{ADP}}{ }^{\lim } \\
(\mathrm{nm})\end{array}$ & $\begin{array}{l}\sigma_{2}^{\lim a} \\
(\mathbf{G M})\end{array}$ & $\begin{array}{c}\lambda_{\mathrm{ADP}}{ }^{\max } \\
(\mathbf{n m})\end{array}$ & $\begin{array}{l}\sigma_{2}^{\max a} \\
(\mathrm{GM})\end{array}$ & $\begin{array}{c}\sigma_{2}{ }^{\lim . \Phi_{\mathrm{F}} b} \\
(\mathrm{GM})\end{array}$ & $\begin{array}{c}\sigma_{2}{ }^{\max } \cdot \Phi_{\mathrm{F}}{ }^{b} \\
(\mathrm{GM})\end{array}$ & $\begin{array}{l}\sigma_{2}{ }^{\lim } \cdot \Phi_{\mathrm{F}} / \mathbf{M}^{c} \\
(\mathrm{GM} / \mathrm{g} \cdot \mathrm{mol})\end{array}$ & $\begin{array}{l}\text { EF1 }{ }^{d} \\
(\mathrm{GM})\end{array}$ & $\begin{array}{c}\sigma_{2}{ }^{\lim } \cdot \Phi_{\Delta}{ }^{e} \\
(\mathbf{G M})\end{array}$ & $\begin{array}{l}\sigma_{2}{ }^{\lim } \cdot \Phi_{\Delta} / \mathbf{M}^{f} \\
(\mathrm{GM} / \mathrm{g} \cdot \mathrm{mol})\end{array}$ & $\begin{array}{l}\text { EF2 }{ }^{g} \\
(G M)\end{array}$ & Refs \\
\hline $\mathrm{ZnPc}$ & 820 & 200 & 880 & $150^{c}$ & 52 & 39 & 0.090 & 1.00 & 122 & 0.212 & 1.00 & [33a] \\
\hline $\mathrm{ZnI}_{4} \mathrm{Pc}$ & 820 & 90 & 880 & 40 & 10 & 4 & 0.009 & 0.19 & & - & & This work \\
\hline $2 a$ & 790 & 380 & - & - & 76 & - & 0.042 & 1.46 & 266 & 0.147 & 2.18 & {$[11 \mathrm{~b}]$} \\
\hline $2 b$ & 790 & 200 & - & - & 22 & - & 0.007 & 0.69 & 118 & 0.039 & 0.97 & {$[11 \mathrm{~b}]$} \\
\hline $2 c$ & 790 & 290 & - & - & 38 & - & 0.012 & 0.73 & 162 & 0.052 & 1.33 & {$[11 \mathrm{~b}]$} \\
\hline $3 b$ & 830 & 330 & 880 & 160 & 109 & 53 & 0.060 & 2.10 & 188 & 0.104 & 1.54 & [18] \\
\hline 4 & 860 & 160 & 880 & 140 & 37 & 32 & 0.020 & 0.71 & 96 & 0.053 & 0.79 & [18] \\
\hline 5 & 830 & 510 & 880 & 240 & 189 & 89 & 0.088 & 3.63 & 275 & 0.128 & 2.26 & [18] \\
\hline $6 a$ & 840 & 1010 & 880 & 630 & 293 & 189 & 0.165 & 5.83 & 606 & 0.341 & 4.97 & This work \\
\hline $6 b$ & 840 & 1640 & 880 (sh) & 670 & 525 & 214 & 0.155 & 10.10 & 836 & 0.248 & 6.86 & This work \\
\hline $6 c$ & 840 & 1880 & 880 (sh) & 670 & 677 & 241 & 0.103 & 13.02 & 996 & 0.151 & 8.17 & This work \\
\hline 7 & 840 & 900 & $870(\mathrm{sh})$ & 420 & 270 & 126 & 0.124 & 5.19 & - & - & - & This work \\
\hline
\end{tabular}

${ }^{a}$ Maxima of the intrinsic 2PA cross-sections measured in THF solutions by TPEF in the femtosecond regime; a fully quadratic dependence of the fluorescence intensity on the excitation power is observed. ${ }^{\mathrm{b}}$ Two-photon brightness (or $2 \mathrm{P}$ action cross-section) at the $2 \mathrm{PA}$ maximum.$^{[35] \mathrm{c}}$ Two-photon brightness corrected for the molecular weight (M). ${ }^{\mathrm{d}}$ Enhancement factor for 2PA imaging relative to ZnPc at the limiting 2PA wavelength; EF1 $=\sigma_{2}{ }^{\text {lim }} . \Phi_{\mathrm{F}} / 52 .{ }^{11 \mathrm{~b}]}$ e Two-photon efficiency for PDT. ${ }^{\mathrm{f}}$ Two-photon efficiency for PDT corrected for the molecular weight. ${ }^{\mathrm{g}}$ Enhancement factor for $2 \mathrm{P}-\mathrm{PDT}$ relative to $\mathbf{Z n P c}$ at the limiting $2 \mathrm{PA}$ wavelength; EF2 $=\sigma_{2}{ }^{\text {lim }} . \Phi_{\Delta} / 122 .{ }^{[1 \mathrm{~b}]}$ 
Regardless the exact origin of 2PA, the cross-sections of all these maxima appear to strongly depend on the chemical composition of the peripheral dendrons. As expected, according to Figure 4 , the existence of an extended $\pi$-system on the dendrons seems to induce larger $\sigma_{2}$ values among 6a-c and 7. In this respect, a great improvement is presently observed for all these compounds when the $\sigma_{2}{ }^{\lim }$ or $\sigma_{2}{ }^{\max }$ values are compared to those previously found for phthalocyanines 3-5. The largest of them was obtained for 5 (510/240 GM) and remains two to three times lower than those of 6a-c or $\mathbf{7}$ (Table 2). Then, an even larger improvement in $\sigma_{2}$ values is obtained relative to the porphyrin-based dendrimers $\mathbf{2 a - c}$. Furthermore, compared to these molecules, the 2PA maxima are significantly shifted to the red for the phthalocyanine derivatives 6a-c, which is also beneficial for most bio-oriented applications. ${ }^{[36]}$ Given that similar dendrons are present in 6a-c and $\mathbf{2 a - c}$, part of this enhancement can be attributed to the change in the central tetrapyrrolic unit, while the improvement stated for $\mathbf{6 a - c}$ and $\mathbf{7}$ over $\mathbf{3 b} \mathbf{b}$ 5 can be traced back to the existence of a better conjugation between the peripheral dendrons and the central ring. To understand better the structural dependence of these nonlinear absorptions, we have normalized them by dividing the 2PA cross-sections $\sigma_{2}$ by the square of the effective number of $\pi$-electrons $\left(N_{\text {eff }}^{2}\right)$. These $\sigma_{2} / N_{\text {eff }}^{2}$ values are figures of merit allowing to take into consideration the influence of the different numbers of $\pi$-electrons present in each compound on $\sigma_{2}$ values (See ESI, Table S1). They should permit a better comparison between them regardless of their different $\pi$-manifolds. ${ }^{[37] 4}$ According to these figures, the lower generation dendrimer 6a presents the optimal 2PA response among the new dendrimers, with a ranking in the order: $\mathbf{6 a}>\mathbf{6 b}>\mathbf{6 c} \approx 7$. In other words, the increase in $\sigma_{2}$ when progressing from 6a to $\mathbf{6 c}$ in not proportional to the number of the most NLO-active electrons in these compounds. Furthermore, all the new compounds presently isolated appear to perform better, per active electron, then $\mathbf{2 a - c}$ and $\mathbf{3 b - 5}$, pointing to a better structural design for the new dendrimers 6a-c.

Finally, the two-photon action cross-section $\left(\sigma_{2} . \Phi_{\mathrm{F}}\right)$, also called two-photon brightness, provides information about the suitability of two-photon absorbers to be used for fluorescence imaging (Table 3). ${ }^{[35]}$ Likewise, the product $\sigma_{2} . \Phi_{\Delta}$ informs about their suitability to perform two-photon PDT. In line with the enhancement factors (EF1 and EF2) presently derived (Table 2), the significantly larger values found for 6a-c outline a clear advantage for these dendrimers

\footnotetext{
${ }^{4}$ The effective numbers of $\pi$-electrons ( $N_{\text {eff }}$ values) were derived for each compound considering peripheral dendrons disconnected from the central phthalocyanine core (Table S1, ESI).
} 
relative to 3-5 or to the porphyrin-based dendrimers 2a-c for these tasks and highlight the existence of a dendrimeric effect, which makes it possible to boost their performances upon moving to higher generations. Then, considering figures of merit, which correct these values for the molecular weight (or for the volume) of the molecules (Table 2), we see that the advantage in favor of the higher generations is lost. This indicates that the increase in performances does not scale anymore with the increase in their size, in line with the statement made above for effective $\pi$-electrons. ${ }^{5}$ However, while comparison with other phthalocyaninebased photosensitizers is not always easy due to the lack of comparable data in the literature, ${ }^{[19 a}$, 19b, 38] the photonic performances presently determined for 6a-c and 7 are clearly better than those of most of the systems currently in use for cancer treatment or imaging. ${ }^{[19 b]}$

DFT calculations and molecular dynamics (MD) simulations were undertaken to gain more insight about the electronic and steric impact of the peripheral dendrons on the properties of the central macrocycle in these new dendrimers.
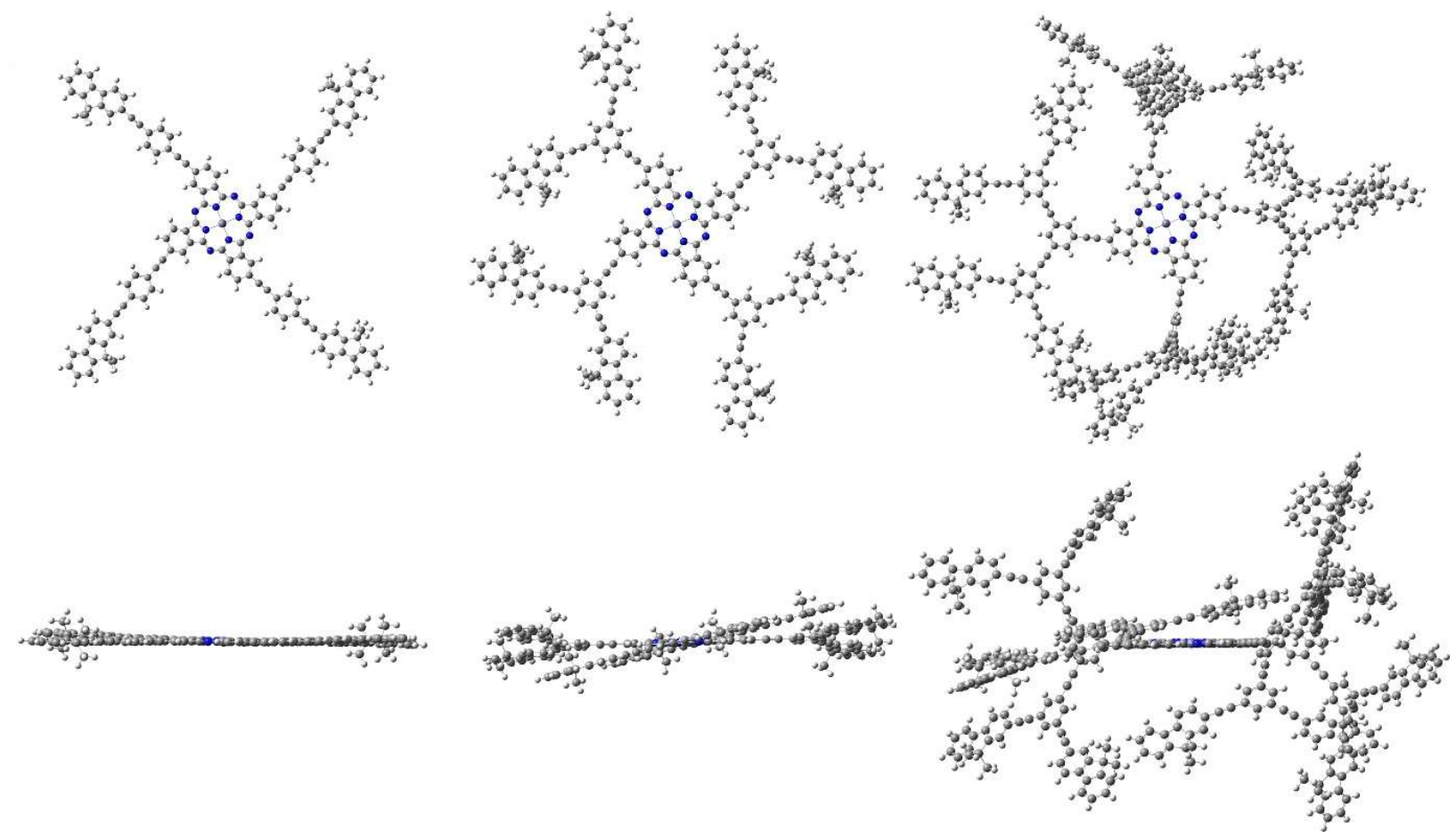

Figure 5. Optimized geometry for $\mathbf{7}^{\prime}, \mathbf{6 b}^{\prime}$ and $\mathbf{6} \mathbf{c}^{\prime}$ dendrimers (view from above and side view).

Following geometry optimization in vacuum, the electronic structure of 6a-c and 7 were computed using the model compounds 6a'-c' and 7' in which the $n$-butyl groups on the 9 position of the 2-fluorenyl groups have been replaced by methyl groups. These molecular

\footnotetext{
${ }^{5}$ The molecular mass and volume of molecules are usually related through the relative constancy of their volumic mass (or density).
} 
models correspond to tetra-substituted $\beta$-regioisomers symmetrically functionalized in positions 2, 9, 16 and 23. After geometry optimization, the molecules 6a' and 7' (Figures S19 and 5) appear completely flat with the fluorene moieties and the phthalocyanine core (and also the phenyl linkers for 7') lying in the same plane. As stated for 7', the various possible orientations of the fluorene fragments in this plane do not appear to affect significantly the energy ordering of the frontier molecular orbitals (FMOs). As shown on Figure 5, the fluorene fragments cannot all remain in the phthalocyanine plane for higher generations due to steric congestion. Thus, for $\mathbf{6} \mathbf{b}^{\prime}$, the arms start twisting out of this plane $\left(9.57^{\circ}\right)$. This effect is much more marked with $\mathbf{6 c}$ ', for which the molecule adopts a 3D geometry with fluorene fragments distributed all around the phthalocyanine core. From these studies, only 6a'-b' and 7' appear liable to undergo stacking interactions via their phthalocyanine core in solution, although the presence of the various $n$-butyl chains in the real molecules might interfere, especially in $6 \mathbf{6}$ and 7. The HOMOs of $\mathbf{6 a}^{\prime}$ ' and 7' in these conformers are completely delocalized over the whole molecule (Figure 6), in line with a good electronic conjugation though the parasubstituted phenyl linkers in 7. In contrast, the HOMOs of $\mathbf{6} \mathbf{b}^{\prime}$ and $\mathbf{6} \mathbf{c}^{\prime}$ only extend to the first phenyl ring of the dendrons, indicating that the meta-substitution hinders conjugation, which is possibly also disfavored by the less coplanar conformations adopted by the dendrons in these molecules. Then, for all compounds, the degenerate LUMO and LUMO+1 set is essentially located on the phthalocyanine core. In line with the blue shift experimentally observed for the Q-band between 7, 6a and $\mathbf{6 b} / \mathbf{6 c}$, the HOMO/LUMO gap increases from $2.00 \mathrm{eV}$ for $\mathbf{7}$ ' to 2.03 $\mathrm{eV}$ for $\mathbf{6} \mathbf{a}^{\prime}$ to $2.08 \mathrm{eV}$ for $\mathbf{6} \mathbf{b}^{\prime}$ and finally to $2.14 \mathrm{eV}$ for $\mathbf{6} \mathbf{c}^{\prime}$. However, the increase in gap predicted by the calculations between $\mathbf{6} \mathbf{b}^{\prime}$ and $\mathbf{6} \mathbf{c}^{\prime}$ does not reflect the Q-band energy experimentally found for $\mathbf{6 b}$ and $\mathbf{6 c}$. Actually, the gaps predicted for $\mathbf{6 b}$ ' and $\mathbf{6 c} \mathbf{c}^{\mathbf{2}}$ recall values computed for phthalocyanines such as $\mathbf{3 b}(2.11 \mathrm{eV})$ or $\mathbf{5}(2.14 \mathrm{eV})$, presenting four unconjugated arms at their periphery. Thus, the significantly lower gaps calculated for $\mathbf{6 a} \mathbf{\prime}$ ' and 7' likely originate from existence of $\pi$-conjugation between the peripheral arms and the central phthalocyanine core and from the extended $\pi$-conjugation within these peripheral arms, at least compared to 6b' and 6c'. 

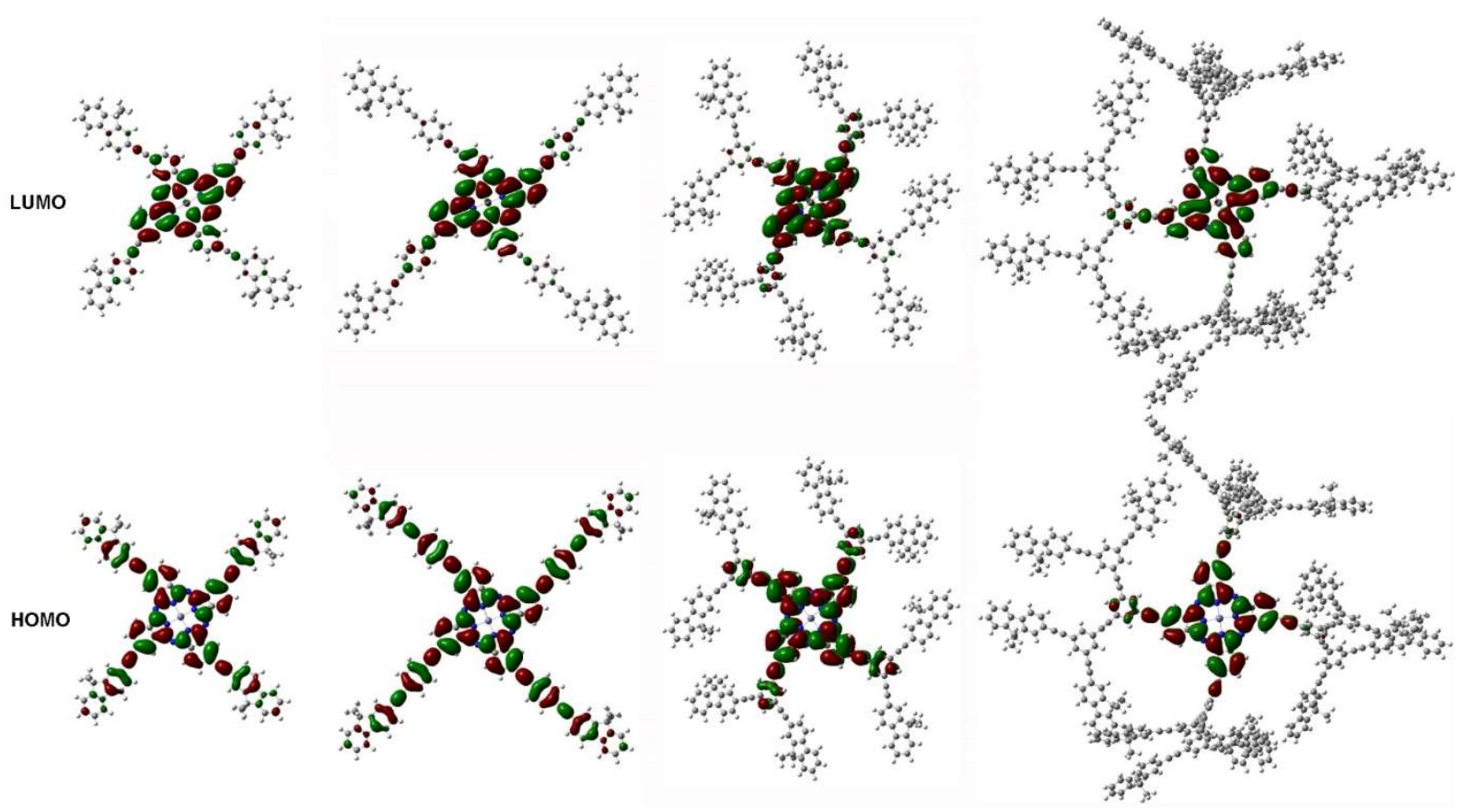

Figure 6. Frontier molecular orbitals (bottom: HOMO, top: LUMO), of dendrimers 6a'-c' and 7' (isocontour value of $\left.0.01\left[\mathrm{e} \mathrm{bohr}^{-3}\right]^{1 / 2}\right)$.

Time-dependent density functional theory (TD-DFT) calculations were next undertaken on 6' ' and 7' to assess the nature of the main electronic transitions experimentally observed for their real counterparts $6 \mathbf{a}$ and $7 .{ }^{6}$ In this respect, the simulated spectra (Figure S20, ESI) display a set of nearly degenerate transitions in the $670-680 \mathrm{~nm}$ range corresponding to $\mathrm{HOMO} \rightarrow$ LUMO and $\mathrm{HOMO} \rightarrow$ LUMO +1 excitations with high oscillator strengths, formally corresponding to those of the Q-Band. ${ }^{7[39]}$ Then, another set of allowed $\pi \rightarrow \pi^{*}$ transitions partly centered on the phthalocyanine core and formally giving rise to the B-band ${ }^{[39]}$ is computed at about $380 \mathrm{~nm}$ for $\mathbf{6} \mathbf{a}^{\prime}$ and around $409-433 \mathrm{~nm}$ for 7 '. Due to the intimate admixture of fluoreneand phthalocyanine-based $\pi$ and $\pi *$ MOs, all these $\pi \rightarrow \pi^{*}$ transitions present some chargetransfer $(\mathrm{CT})$ character between the peripheral dendrons and the central core and none of them remains strictly localized on the tetrapyrrolic core. Finally, several moderate-to-intenselyallowed $\pi \rightarrow \pi^{*}$ transitions, heavily weighted on the peripheral dendrons, are computed between 380 and $359 \mathrm{~nm}$ for $\mathbf{6} \mathbf{a}^{\prime}$ and between 409 and $373 \mathrm{~nm}$ for $\mathbf{7}$ '. These excitations, which again present various degree of CT character with the central phthalocyanine core, give rise to an

\footnotetext{
${ }^{6}$ Such calculations were found to be too time and resource consuming for the higher generation dendrimers $\mathbf{6 b}$ ' and $\mathbf{6 c}$.

${ }^{7}$ Given that the vibronic splitting of that band is not reproduced by TD-DFT calculations, we have only listed the most intense vibronic band in Table 3. Evidently, this will contribute to decrease the match between the experimental and simulated spectra.
} 
intense band located on the high energy side of the B-band, recalling the band which was attributed to arm-centered transitions on the absorption spectra of $\mathbf{6 a}$ and 7 (Figure 2). Interestingly, two moderately allowed excitations are also found at wavelengths between those of the Q- and B-bands, a spectral range in which no transitions were experimentally detected. These transitions actually correspond to $\pi_{\mathrm{Flu}} \rightarrow \pi^{*} \mathrm{Pc}$ CT processes in 6a' and 7'. We believe that these transitions take place at slightly different energies in the real molecules $\mathbf{6 a}$ and $\mathbf{7}$, and might therefore either be hidden beneath the vibronic sub-bands experimentally observed on the high energy-side the Q-band or could correspond to the shoulder observed on the low energy-side of the B-Band. Similar transitions were previously also calculated (and not observed) for the $\mathrm{Zn}$ (II) phthalocyanine complex 5. $^{[18]}$ Notably, other excitations of the same kind, but with negligible oscillator strength, are also computed in the same energy range. These might correspond to the forbidden $g-g$ states giving rise to 2PA (see above). Considering that the experimental measurements were obtained with mixtures of stereoisomers and that neither the dielectric constant of the solvent nor the vibronic coupling were taken in consideration during the calculations, the agreement between the simulated and experimental UV-Vis spectra can be considered as correct for the main transitions.

Finally, in order to obtain additional insight about possible geometrical issues related to the dendritic structures, the conformations of the real dendrimers 6a-c and 7 (with butyl chains) have been simulated by molecular dynamics (MD) in vacuum and in THF solution. MD simulations run for $\mathbf{6 a}$ and 7 , with or without THF over $4 \mathrm{~ns}$, reveal that these molecules remain almost flat during all the simulation time, undergoing only flapping or twisting of the peripheral fluorene arms around their initial position (Figure 7, SI Video S1 and S2). Now, when such molecules are considered in the same volume, they show some interactions through the extended arms but no tendency to stack (SI Video S3). On contrary, in $\mathrm{CH}_{2} \mathrm{Cl}_{2}$ (starting from the same positions), these molecules stack readily and form well-eclipsed dimers with a good overlap of their phthalocyanine cores (SI Video S4), which is in line with their insolubility in dichloromethane.

Considering the conformational evolution of $\mathbf{6 b}$ in solution, a related statement can be made; the central phthalocyanine core also remains almost coplanar during the simulation, while extensive flapping and twisting of the fluorenyl groups within their peripheral arms are observed. This motion is also accompanied by large rotations of each dendron around the triple bond linking its first phenyl group to the central core (Figure 7, SI Video S5). In this compound $\mathbf{6 b}$, the extended arms are well isolated and can freely rotate and no interactions between the 
neighbouring arms through the butyl chains or the fluorene fragments is observed. Also, notably, a conformation with the four peripheral dendrons oriented perpendicular to the phthalocyanine core is never observed among the most stable ones for this molecule. Then, in contrast to $\mathbf{6 b}$, the dendrimer $\mathbf{6 c}$ appears much more flexible in solution and strong interactions between the neighbouring arms can be observed. During the simulation the large dendrons rotate and curve to eventually interact in an intramolecular way through $\pi$-stacking interactions. This evolution contributes to flattening the molecule from a ball-shaped conformation into transitory a donut-like shaped conformation (Figure 7, bottom, SI Video S6). The bending and the clustering of the fluorene arms is driven here by strong intramolecular $\pi$ - $\pi^{*}$ interactions between fluorene fragments but also between fluorene fragments and phenyl rings of neighbouring arms (see white circles, on Figure 7). ${ }^{8}$ In spite of this flattening process, the dendron shell remains too bulky to allow any intermolecular $\pi$ - $\pi$ stacking process to occur between phthalocyanine cores (SI Video S7). ${ }^{9}$

These results confirm that, at concentration as high as $7.87410^{-3} \mathrm{~mol} . \mathrm{L}^{-1}$, these dendrimers remain well isolated in THF solution. In addition, the Brownian "paddlewheel" motions of the dendrons evidenced by MD certainly contributes to disfavour spontaneous aggregation for the largest dendrimers $(\mathbf{6 b}-\mathbf{c})$ it may also restrict direct access to the central $\mathrm{Zn}$ (II) phthalocyanine unit for molecules localized in equatorial positions. A contrario, the overall disk-shaped or donut-shaped conformation eventually adopted by $\mathbf{6 b}$ and $\mathbf{6 c}$, respectively, can restore an easier axial access to this unit, especially for small molecules (such as oxygen). Ultimately, these nearly coplanar conformations adopted in solution, will contribute to optimize the electronic interactions between the peripheral dendrons and the central phthalocyanine core (via $\pi$-conjugation) and might possibly also reinforce the magnetic deshielding of its protons at the same time.

\footnotetext{
${ }^{8}$ In the absence of solvent, this dendrimer $\mathbf{6 c}$ adopts a pocket-like conformation, with the arms all aggregated on one side of the phthalocyanine core, showing the high flexibility of this molecule (Figure S21).

${ }^{9}$ Stacking distances of $24.6 \pm 3.5 \AA$ are usually observed between phthalocyanine cores in MD simulations with the large dendrimer $\mathrm{G}_{3}$. Note that concentrations used during these simulations $\left(7.87410^{-3} \mathrm{M}\right)$ are much higher than the experimental ones.
} 

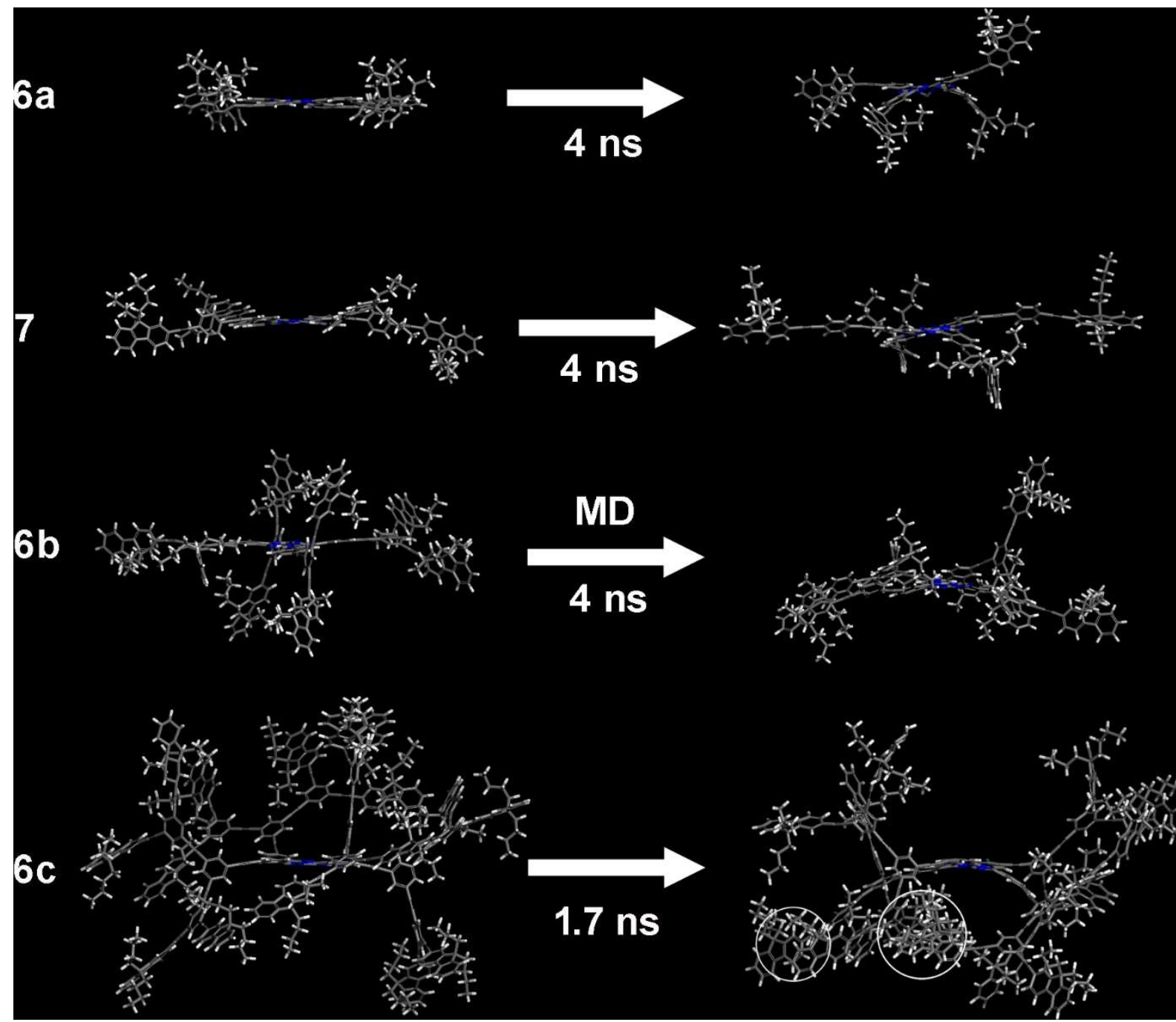

Figure 7. Left: Starting configuration of one molecule of 6a, 7, 6b and 6c. Right: molecular configuration after energy relaxation and 4ns simulation in NVT ensemble (fixed Number of molecules, Volume and Temperature) in a unit cell of $\mathrm{a}=75 \AA, \mathrm{b}=75 \AA, \mathrm{c}=75 \AA, \alpha=\beta=\gamma=90^{\circ}$ filled with THF molecules (density = 1). For large 6c, the image was caught after $1.7 \mathrm{~ns}$. 
Table 3. Computed (B3LYP /6-31G*/LANL2DZ) values (nm). Energy and composition of the first singlet excited states (wavelength, oscillator strength $f$, transition percentage).

\begin{tabular}{|c|c|c|c|c|}
\hline \multirow{2}{*}{$\begin{array}{l}\text { Real/Model } \\
\text { Cpnds }\end{array}$} & \multirow{2}{*}{$\begin{array}{c}\text { Observed Transitions for Real } \\
\text { Cpnds }{ }^{a, b} \\
\lambda_{\max }[\varepsilon \max ]\end{array}$} & \multicolumn{3}{|c|}{ Calculated Transitions for Model Cpnds } \\
\hline & & $\begin{array}{c}\text { Energy }^{a, c} \\
\lambda_{\max }[f]\end{array}$ & Composition & $\begin{array}{c}\text { Major } \\
\text { Assignment }\end{array}$ \\
\hline \multirow[t]{16}{*}{ 6a/6a' } & $697[398]^{d}$ & $666[0.96]$ & $\mathrm{H} \rightarrow \mathrm{L}(95 \%)$ & $\pi^{*} \leftarrow \pi(\mathrm{Q}-$ Band $)$ \\
\hline & $628\left[^{669.2]^{d}}\right.$ & $667[0.96]$ & $\mathrm{H} \rightarrow \mathrm{L}+1(95 \%)$ & \\
\hline & l & $556[0.30]$ & $\mathrm{H}-1 \rightarrow \mathrm{L}(90 \%)$ & $\pi^{*} \mathrm{Pc}_{\mathrm{c}} \leftarrow \pi \mathrm{Flu}$ \\
\hline & & $556[0.30]$ & $H-1 \rightarrow L+1(90 \%)$ & \\
\hline & & $505[0.28]$ & $\mathrm{H}-4 \rightarrow \mathrm{L}(93 \%)$ & \\
\hline & & $505[0.28]$ & $\mathrm{H}-4 \rightarrow \mathrm{L}+1(93 \%)$ & \\
\hline & $380(\mathrm{sh})[122]$ & $384[0.28]$ & $\begin{array}{l}\mathrm{H}-5 \rightarrow \mathrm{L}(30 \%) \\
\mathrm{H}-5 \rightarrow \mathrm{L}+1(34 \%)\end{array}$ & $\pi^{*} \leftarrow \pi(\mathrm{B}-\mathrm{Band})$ \\
\hline & & $384[0.29]$ & $\begin{array}{l}\mathrm{H}-5 \rightarrow \mathrm{L}(33 \%), \\
\mathrm{H}-5 \rightarrow \mathrm{L}+1(29 \%)\end{array}$ & \\
\hline & & $380[0.74]$ & $\mathrm{H} \rightarrow \mathrm{L}+4(56 \%)$ & $\pi^{*} \mathrm{Flu} \leftarrow \pi \mathrm{Pc}$ \\
\hline & & $380[0.74]$ & $\mathrm{H} \rightarrow \mathrm{L}+5(57 \%)$ & \\
\hline & & $371[0.24]$ & $\mathrm{H}-8 \rightarrow \mathrm{L}(67 \%)$ & $\pi^{*} \mathrm{Pc} \leftarrow \pi \mathrm{Flu}$ \\
\hline & & $371[0.25]$ & $\mathrm{H}-8 \rightarrow \mathrm{L}+1(67 \%)$ & \\
\hline & 338 [179] & $359[1.21]$ & $\mathrm{H}-2 \rightarrow \mathrm{L}+2(62 \%)$ & $\pi^{*} \mathrm{Flu}+\mathrm{Pc}_{\mathrm{c}} \leftarrow \pi \mathrm{Flu}$ \\
\hline & & $359[1.21]$ & $\mathrm{H}-3 \rightarrow \mathrm{L}+2(62 \%)$ & \\
\hline & & $346[0.22]$ & $H-21 \rightarrow L+1(55 \%)$ & $\pi^{*} \leftarrow \pi(\mathrm{N}-\text { Band })^{e}$ \\
\hline & & $346[0.23]$ & $\mathrm{H}-21 \rightarrow \mathrm{L}(57 \%)$ & \\
\hline \multirow[t]{16}{*}{$7 / 7^{\prime}$} & $696[373]^{d}$ & $681[1.20]$ & $\mathrm{H} \rightarrow \mathrm{L}(91 \%)$ & $\pi^{*} \leftarrow \pi(\mathrm{Q}-$ Band $)$ \\
\hline & $627[69.2]^{d}$ & $680[1.23]$ & $\mathrm{H} \rightarrow \mathrm{L}+1(91 \%)$ & \\
\hline & l & $596[0.33]$ & $\mathrm{H}-1 \rightarrow \mathrm{L}(98 \%)$ & $\pi^{*} \mathrm{Pc}_{\mathrm{c}} \leftarrow \pi \mathrm{Flu}$ \\
\hline & & $596[0.31]$ & $\mathrm{H}-1 \rightarrow \mathrm{L}+1(98 \%)$ & \\
\hline & & $549[0.23]$ & $\mathrm{H}-4 \rightarrow \mathrm{L}(94 \%)$ & \\
\hline & & $548[0.23]$ & $\mathrm{H}-4 \rightarrow \mathrm{L}+1(94 \%)$ & \\
\hline & $388(\mathrm{sh})[153]$ & $433[0.54]$ & $\mathrm{H}-8 \rightarrow \mathrm{L}(87 \%)$ & $\pi^{*} \leftarrow \pi($ B-Band $)$ \\
\hline & & $433[0.55]$ & $\mathrm{H}-8 \rightarrow \mathrm{L}+1(87 \%)$ & \\
\hline & & 409 [1.26] & $\mathrm{H} \rightarrow \mathrm{L}+4(82 \%)$ & $\pi^{*} \mathrm{Flu} \leftarrow \pi \mathrm{Pc}$ \\
\hline & & $409[1.35]$ & $\mathrm{H} \rightarrow \mathrm{L}+5(82 \%)$ & \\
\hline & 356 [289] & 393 [1.63] & $\mathrm{H}-2 \rightarrow \mathrm{L}+2(70 \%)$ & $\pi^{*} \mathrm{Pc}_{\mathrm{c}} \leftarrow \pi \mathrm{Flu}$ \\
\hline & & 393 [1.78] & $\mathrm{H}-3 \rightarrow \mathrm{L}+2(70 \%)$ & \\
\hline & & 373 [1.30] & $\mathrm{H}-2 \rightarrow \mathrm{L}+2$ (14\%), & \\
\hline & & & $\mathrm{H}-2 \rightarrow \mathrm{L}+3(51 \%)$ & \\
\hline & & 373 [1.38] & $H-3 \rightarrow L+2(14 \%)$ & \\
\hline & & & $H-3 \rightarrow L+3(51 \%)$ & \\
\hline
\end{tabular}

$\bar{a}$ in nm. ${ }^{b}$ Experimental absorption coefficient (in $10^{3} \mathrm{M}^{-1} \cdot \mathrm{cm}^{-1}$ ). ${ }^{c}$ Computed oscillator strength. ${ }^{d}$ Vibronic sibbands (not modelled by computations). ${ }^{\mathrm{e}}$ Tentative assignment.

\section{- CONCLUSIONS}

In this work we investigate the impact of changing the core in tetrapyrrolic Dendrimers. So, we have described herein the synthesis and characterization of a series of new dendrimers (6a-c and 7) presenting "carbon-rich" dendrons conjugated with a central Zn(II) phthalocyanine core and having four to sixteen 9,9-dibutyl-2-fluorenyl groups at their periphery. These new dendrimers were actually isolated as mixtures of tetra-substituted $\beta$-regioisomers. Thanks to the 
presence of numerous $n$-butyl side-chains, but also thanks to the rigidity of the peripheral dendrons, which contribute (upon randomly rotating) to disfavor stacking in solution for the higher generations below $10^{-3} \mathrm{M}$, a good solubility is observed for all these dendrimers in organic solvents. In terms of linear photophysical properties, these dendrimers exhibit a high luminescence, with quantum yields up to $36 \%$. These yields depend on the dendron structure (e.g. compare 6a and 7) and also, for a given structure, on the generation considered (e.g. compare 6a-c) increasing for higher generations (i.e. from $\mathrm{G}_{0}$ to $\mathrm{G}_{2}$ ). As previously stated for related porphyrin- and phthalocyanine-based dendrimers, a very efficient energy transfer from the peripheral fluorenyl units toward the central tetrapyrrolic core allows these photochromic systems to efficiently "harvest" energy from UV-visible light and to funnel it toward the central phthalocyanine $\mathrm{Zn}(\mathrm{II})$ complex. There, it can be re-emitted or re-used to photosensitize molecular oxygen with good efficiency, making all these new dendrimers appealing photosensitizers. In this respect, MD calculations reveal that a "donut-shaped" structure is adopted in solution for the higher generation members which allows axial access to the central tetrapyrrolic unit to small molecules such as oxygen. Thus, albeit the quantum yields of 6a-c or 7 for singlet oxygen formation are slightly lower (around 51-55\%) than that of the zinc(II) phthalocyanine complex $(\mathbf{Z n P c})$ often used as reference, these new photosensitizers retain a sufficient ability to produce cytotoxic oxygen for envisioning applications in PDT, especially if the light-harvesting capabilities of the peripheral dendrons is exploited in the choice of the excitation wavelength. In this respect and considering their relative one-photon brightness, 6ac and 7 appear better suited for performing simultaneously one-photon imaging and PDT than the related systems previously studied, such as phthalocyanine-based dendrimers featuring nonconjugated fluorenyl groups at their periphery (3-5) or series of analogous mesotetraphenylporphyrin-cored dendrimers (2a-c) previously developed. More remarkably and in line with our expectations, 6a-c and 7 function as efficient two-photon absorbers. Thanks to their peripheral dendrons featuring an extended $\pi$-manifold conjugated to the central tetrapyrrolic core, these nonlinear chromophores, exhibit fairly large intrinsic 2PA crosssections (up to $1880 \mathrm{GM}$ for $\mathbf{6 c}$ ), which increase with the size of their peripheral dendrons. The values presently found are significantly larger than those previously determined for 3-5 and for 2a-c. This in turn induces better figures of merit for two-photon imaging and for oxygen sensitization, making these new systems more appealing than the former ones for applications in two-photon bioimaging or PDT. However, when their size or the number of active $\pi$ electrons $\left(N_{\text {eff }}\right)$ is taken into consideration for evaluating their nonlinear absorption properties, the dendrimers of higher generation appear to be not as efficient as some of the smaller systems,

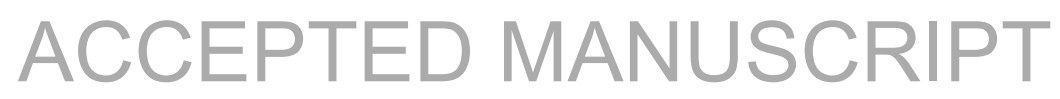


leaving some room for further improvement. More generally, based on the available data regarding the relevant photonic performances, these new dendrimers stand the comparison with other phthalocyanine-based systems currently used as one- or two-photon photosensitizers in cancer therapy. ${ }^{[19 b]}$ These first results are therefore very encouraging to explore further their applied potential in PDT, theranostic or related medical uses. ${ }^{[19 \mathrm{~d}, 19 \mathrm{e}, 40]}$ Work along these lines is currently in progress in our group.

\section{口 EXPERIMENTAL SECTION}

Supplementary tables and figures are provided in the Supporting Information: ${ }^{1} \mathrm{H}$ NMR and ${ }^{13} \mathrm{C}$ NMR characterization of all new compounds, selected FMO plots, TD-DFT and MD results, cartesian coordinate of the model compounds, two-photon absorption data for 6a-c and 7. Seven video clips representing selected MD simulations.

Materials. Compounds were purified by chromatography on silica gel using different mixtures of eluents as specified. Unless otherwise stated, all solvents used in reactions were distilled using common purification protocols, except DMF and ${ }^{i} \operatorname{Pr}_{2} \mathrm{NH}$ which were dried on molecular sieves $(3 \AA)$.. ${ }^{1} \mathrm{H}$ and ${ }^{13} \mathrm{C}$ NMR spectra were recorded on Bruker Ascend 400 and 500 at $298 \mathrm{~K}$. The chemical shifts are referenced to internal tetramethylsilane (TMS). Highresolution mass spectra were recorded on different spectrometers: a Bruker MicrOTOF-Q II, a Thermo Fisher Scientific Q-Exactive in ESI positive mode and a Bruker Ultraflex III MALDI Spectrometer at CRMPO (Centre Regional de Mesures Physiques de l'Ouest) in Rennes. Reagents were purchased from commercial suppliers and used as received. The dendron

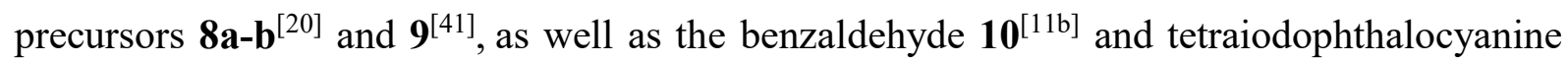
complex $\mathbf{Z n I}_{4} \mathbf{P c}^{[21]}$ were obtained as previously described in the literature.

Synthesis. General Procedure for 4-((9,9-Dibutyl-9H-fluoren-2-yl)ethynyl) phthalonitrile (12): In a Schlenk tube, to a mixture of ethynyfluorenyl 8a (227 mg, $0.75 \mathrm{mmol}$, 1.5 eq), 4-iodophthalonitrile (127 mg, $0.5 \mathrm{mmol}, 1 \mathrm{eq}), \mathrm{Pd}\left(\mathrm{PPh}_{3}\right)_{2} \mathrm{Cl}_{2}$ (3.5 mg, $0.005 \mathrm{mmol}, 1 \%$ eq) and $\mathrm{CuI}\left(0.6 \mathrm{mg}, 0.003 \mathrm{mmol}, 0.6 \%\right.$ eq), were added THF $(2 \mathrm{~mL})$ and $\mathrm{NEt}_{3}(8 \mathrm{~mL})$ under argon. The mixture was degassed and stirred for $16 \mathrm{~h}$ at room temperature. After being evaporated, the residue was filtered and extracted three times with $\mathrm{CH}_{2} \mathrm{Cl}_{2}$. The organic layer was dried with $\mathrm{MgSO}_{4}$, filtered and the solvent was evaporated. The crude product was adsorbed onto silica and further purified by chromatography using heptane $/ \mathrm{CH}_{2} \mathrm{Cl}_{2}(3: 1)$ as eluent, leading to 12 as a white powder (167 mg, 78\% yield). ${ }^{1} \mathbf{H}$ NMR (400 $\left.\mathrm{MHz} \mathrm{CDCl}_{3}\right): \delta=$ 
$7.95(\mathrm{~d}, J=1.6 \mathrm{~Hz}, 1 \mathrm{H}), 7.85\left(\mathrm{dd}, J_{1}=8.2, J_{2}=1.6 \mathrm{~Hz}, 1 \mathrm{H}\right), 7.79(\mathrm{~d}, J=8.2 \mathrm{~Hz}, 1 \mathrm{H}), 7.75-$ $7.69(\mathrm{~m}, 2 \mathrm{H}), 7.57-7.51(\mathrm{~m}, 2 \mathrm{H}), 7.40-7.33(\mathrm{~m}, 3 \mathrm{H}), 2.06-1.93(\mathrm{~m}, 4 \mathrm{H}), 1.09$ (h, J= 7.4 $\mathrm{Hz}, 4 \mathrm{H}), 0.68(\mathrm{t}, J=7.3 \mathrm{~Hz}, 6 \mathrm{H}), 0.64-0.52(\mathrm{~m}, 4 \mathrm{H}) .{ }^{13} \mathbf{C} \mathbf{N M R}\left(101 \mathrm{MHz}, \mathrm{CDCl}_{3}\right): \delta=151.2$, 151.1, 143.1, 139.9, 135.9, 135.4, 133.5, 131.1, 129.7, 128.2, 127.1, 126.3, 123.0, 120.3, 119.9, 116.3, 115.3, 114.8, 114.0, 98.2, 86.0, 55.2, 40.1, 25.9, 23.0, 13.8. FT-IR $\left(\mathrm{cm}^{-1}\right): \bar{v}=3052(\mathrm{w}$,

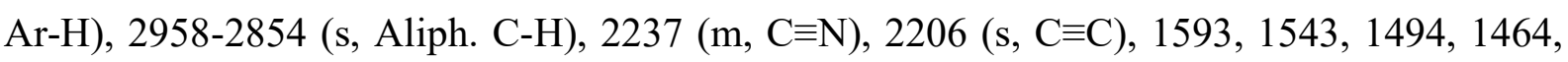
1451, 1419, 1271, 1186, 1086, 940, 894, 842, 780, 741. HRMS-ESI (m/z): $[\mathrm{M}+\mathrm{Na}]^{+}$ $\left(\mathrm{C}_{31} \mathrm{H}_{28} \mathrm{~N}_{2} \mathrm{Na}\right) 451.2151$ (calcd: 451.2145). Anal. Calcd. (\%) for $\mathrm{C}_{31} \mathrm{H}_{28} \mathrm{~N}_{2}$ : C, 86.88; H, 6.59; N, 6.54. Found: C, 87.01; H, 6.44; N, 6.43.

General Procedure for

2,2',2"',2"'-((((5-(2,2-Dibromovinyl)-1,3phenylene)bis(ethyne-2,1-diyl))bis(benzene-5,1,3-triyl))tetrakis(ethyne-2,1-

diyl))tetrakis(9,9-dibutyl-9H-fluorene) (11): In a Schlenk tube, a mixture of $\mathrm{PPh}_{3}$ (144 mg, $0.55 \mathrm{mmol}, 2 \mathrm{eq})$ and $\mathrm{Zn}$ powder $(35.75 \mathrm{~g}, 0.55 \mathrm{mmol}, 2 \mathrm{eq})$ in dry $\mathrm{CH}_{2} \mathrm{Cl}_{2}(20 \mathrm{~mL})$ was cooled to $0{ }^{\circ} \mathrm{C}$ in ice-water bath under argon protection. Then, $\mathrm{CBr}_{4}(182 \mathrm{mg}, 0.55 \mathrm{mmol}, 2 \mathrm{eq})$ was added into Schlenk and the mixture was kept stirring at low temperature for $2 \mathrm{~min}$. The bath was removed and the solution was stirred overnight at room temperature. At last, the mixture was cooled to $0{ }^{\circ} \mathrm{C}$ again and aldehyde 10 (420 mg, $\left.0.27 \mathrm{mmol}, 1 \mathrm{eq}\right)$ in $20 \mathrm{~mL}$ of dry $\mathrm{CH}_{2} \mathrm{Cl}_{2}$ was added under argon, and then, the solution was stirred at room temperature for $4 \mathrm{~h}$. After the evaporation of volatiles, the residue was adsorbed onto silica and further purified by chromatography using petroleum ether/ $\mathrm{CH}_{2} \mathrm{Cl}_{2}(10: 1)$ as eluent system. Intermediate 11 was obtained as a white powder (400 mg, 89\% yield). ${ }^{1} \mathbf{H}$ NMR $\left(400 \mathrm{MHz}, \mathrm{CDCl}_{3}\right) \delta=7.78(\mathrm{~s}, 2 \mathrm{H})$, $7.76-7.66(\mathrm{~m}, 15 \mathrm{H}), 7.57-7.48(\mathrm{~m}, 9 \mathrm{H}), 7.39-7.31(\mathrm{~m}, 12 \mathrm{H}), 2.00(\mathrm{t}, J=8.3 \mathrm{~Hz}, 16 \mathrm{H})$, $1.13-1.05(\mathrm{~m}, 16 \mathrm{H}), 0.70$ (t, $J=7.3 \mathrm{~Hz}, 24 \mathrm{H}), 0.66-0.54(\mathrm{~m}, 16 \mathrm{H})$.

General Procedure for 2,2',2",2'"-((((5-Ethynyl-1,3-phenylene)bis(ethyne-2,1diyl))bis(benzene-1,3,5 triyl))tetrakis(ethyne-2,1-diyl))tetrakis(9,9-dibutyl-9H-fluorene) (8c). In a Schlenk tube, $n$-BuLi $(0.22 \mathrm{~mL}, 0.36 \mathrm{mmol}, 3 \mathrm{eq})$ was added to a cold solution of ${ }^{\mathrm{i}} \mathrm{Pr}_{2} \mathrm{NH}(0.05 \mathrm{~mL}, 0.36 \mathrm{mmol}, 3 \mathrm{eq})$ in dry THF $(3 \mathrm{~mL})$ at $-78{ }^{\circ} \mathrm{C}$. After warming up to room temperature, the mixture was injected dropwise into the solution of compound 11 (202 mg, 0.12 mmol, 1 eq) in dry THF $(7 \mathrm{~mL})$ at $-78^{\circ} \mathrm{C}$ in liquid nitrogen-acetone bath. The mixture was stirred at low temperature for $1 \mathrm{~h}$, then quenched with saturated $\mathrm{NH}_{4} \mathrm{Cl}(5 \mathrm{~mL})$. The reaction allowed warming to room temperature. After evaporation of volatiles, the residue was adsorbed onto silica and further purified by chromatography using a mixture of heptane $/ \mathrm{CH}_{2} \mathrm{Cl}_{2}(20: 1)$ as eluent, showing $8 \mathbf{c}$ as a white powder (146 mg, 81\% yield). ${ }^{1} \mathbf{H}$ NMR (400 MHz, $\left.\mathrm{CDCl}_{3}\right)$ : $\delta$ 
$=7.77(\mathrm{~s}, 2 \mathrm{H}), 7.75-7.67(\mathrm{~m}, 13 \mathrm{H}), 7.65(\mathrm{~s}, 2 \mathrm{H}), 7.57-7.51(\mathrm{~m}, 8 \mathrm{H}), 7.38-7.31(\mathrm{~m}, 12 \mathrm{H})$, $3.16(\mathrm{~s}, 1 \mathrm{H}), 2.00(\mathrm{t}, J=8.3 \mathrm{~Hz}, 16 \mathrm{H}), 1.13-1.06(\mathrm{~m}, 16 \mathrm{H}), 0.69$ (t, $J=7.3 \mathrm{~Hz}, 24 \mathrm{H}), 0.65-$ $0.52(\mathrm{~m}, 16 \mathrm{H}) .{ }^{13} \mathrm{C}$ NMR (101 MHz, $\left.\mathrm{CDCl}_{3}\right): \delta=151.1,150.8,141.9,140.3,135.0,134.7$, 134.4, 133.9, 130.7, 127.7, 126.9, 126.1, 124.4, 123.8, 123.5, 123.1, 122.9, 120.9, 120.1, 119.7, 92.0, 89.3, 88.5, 87.8, 81.9, 55.1, 40.2, 25.9, 23.1, 13.8. FT-IR $\left(\mathrm{cm}^{-1}\right): \bar{v}=3305(\mathrm{w}, \equiv \mathrm{C}-\mathrm{H})$,

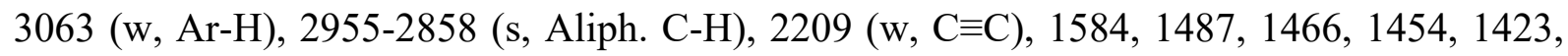
1378, 1298, 1156, 1007, 960, 879, 830, 776, 739. HRMS-ESI (m/z): [M] ${ }^{+}\left(\mathrm{C}_{116} \mathrm{H}_{110}\right) 1502.858$ (calcd: 1502.8602), $[\mathrm{M}+\mathrm{H}]^{+}\left(\mathrm{C}_{116} \mathrm{H}_{111}\right) 1503.867$ (calcd: 1503.86803),

General Procedure for Phthalocyanine 6a (G0): Method I: In a Schlenk tube, to a mixture of tetraiodophthalocyanine $\left(\mathbf{Z n I}_{4} \mathbf{P c}\right)(108,5 \mathrm{mg}, 0.1 \mathrm{mmol}, 1 \mathrm{eq}), 9,9$-dibutyl-2ethynyl-9H-fluorene (8a) (181 mg, $0.6 \mathrm{mmol}, 6 \mathrm{eq}), \mathrm{Pd}\left(\mathrm{PPh}_{3}\right)_{2} \mathrm{Cl}_{2}\left(0.7 \mathrm{mg}, 10^{-3} \mathrm{mmol}, 1 \%\right.$ eq $)$ and $\mathrm{CuI}\left(0.1 \mathrm{mg}, 6 \times 10^{-4} \mathrm{mmol}, 0.6 \%\right.$ eq $)$ were added THF $(5 \mathrm{~mL})$ and $\mathrm{NEt}_{3}(5 \mathrm{~mL})$ under argon. Then, the system was degassed by freeze-pump-thaw twice and stirred overnight at room temperature. After being evaporated, residue was adsorbed onto silica and further purified by chromatography using heptane/diethyl ether (2:1) as eluent system, showing 6a as a green powder (128 mg, $72 \%$ yield).

General Procedure for Phthalocyanine 6a (Go): Method II: A mixture of phthalonitrile 12 (200 mg, $0.47 \mathrm{mmol}, 1 \mathrm{eq}$ ), anhydrous $\mathrm{Zn}(\mathrm{OAc})_{2}$ (42 mg, $0.23 \mathrm{mmol}, 0.5 \mathrm{eq}$ ) and 1,8-diazabicyclo[5.4.0]undec-7-ene (DBU) (5 drops) in degassed $n$-pentanol ( $2 \mathrm{~mL})$ was heated at $160{ }^{\circ} \mathrm{C}$ for $24 \mathrm{~h}$ under argon. The green solution was cooled to room temperature and the suspension was poured into cold methanol. The dark green precipitate was then filtered off and was washed with hot methanol. The crude product was purified by column chromatography on silica gel, using $\mathrm{CH}_{2} \mathrm{Cl}_{2}$ /heptane (1:1) as eluent system and then recrystallized from $\mathrm{MeOH} / \mathrm{CH}_{2} \mathrm{Cl}_{2}$. The zinc complex $6 \mathbf{a}$ was obtained as a green solid (61 mg, 29\% yield).

${ }^{1}$ H NMR (400 MHz, THF- $\left.d_{8}\right): \delta=9.36$ - 9.19 (m, 4H), 9.15 - 8.99 (m, 4H), 8.33 - 8.19 (m, 4H), 7.97 (s, 4H), $7.91-7.75(\mathrm{~m}, 12 \mathrm{H}), 7.56-7.46(\mathrm{~m}, 4 \mathrm{H}), 7.44-7.34(\mathrm{~m}, 8 \mathrm{H}), 2.37-2.15$ $(\mathrm{m}, 16 \mathrm{H}), 1.32-1.20(\mathrm{~m}, 16 \mathrm{H}), 0.92-0.70(\mathrm{~m}, 40 \mathrm{H}) .{ }^{13} \mathbf{C}$ NMR $\left(101 \mathrm{MHz}, \mathrm{THF}-d_{8}\right): \delta=$ 151.2 , 151.1, 142.5, 140.8, 134.0, 131.1, 127.9, 127.2, 126.4, 125.8, 125.7, 124.5, 124.4, 123.1, $122.8,121.5,121.4,120.5,120.2,92.7,91.0,88.0,55.5,40.6,26.5,25.7,23.5,13.7$. FT-IR $\left(\mathrm{cm}^{-1}\right): \bar{v}=3063$ (w, Ar-H), 2956-2861 (s, Aliph. C-H), 2198 (w, C $\left.\equiv \mathrm{C}\right), 1608,1491,1465$, 1450, 1392, 1334, 1298, 1261, 1143, 1087, 1047, 940, 892, 828, 775, 735. HRMS-ESI (m/z): $[\mathrm{M}]^{+} \quad\left(\mathrm{C}_{124} \mathrm{H}_{112} \mathrm{~N}_{8} \mathrm{Zn}\right) \quad 1776.8274 \quad$ (calcd: 1776.8295). Anal. Calcd. (\%) for $\mathrm{C}_{124} \mathrm{H}_{112} \mathrm{~N}_{8} \mathrm{Zn} .2 \mathrm{CH}_{3} \mathrm{OH}$ : C, 82.13; H, 6.68; N, 5.99. Found: C, 81.93; H, 6.40; N, 6.21. 
General Procedure for Phthalocyanine 6a (G0): Phthalocyanine 6b (G1): In a Schlenk tube, to a mixture of tetraiodophthalocyanine $\left(\mathbf{Z n I} \mathbf{I}_{4} \mathbf{P c}\right)(27 \mathrm{mg}, 0.025 \mathrm{mmol}, 1 \mathrm{eq}), \mathbf{8 b}(105 \mathrm{mg}$, $0.15 \mathrm{mmol}, 6 \mathrm{eq}), \mathrm{Pd}\left(\mathrm{PPh}_{3}\right)_{2} \mathrm{Cl}_{2}\left(0.18 \mathrm{mg}, 2.5 \times 10^{-4} \mathrm{mmol}, 1 \%\right.$ eq $)$ and $\mathrm{CuI}(0.029 \mathrm{mg}, 1.5 \mathrm{x}$ $10^{-4} \mathrm{mmol}, 0.6 \%$ eq) were added THF $(5 \mathrm{~mL})$ and $\mathrm{NEt}_{3}(5 \mathrm{~mL})$ under argon. Then, the system was degassed by freeze-pump-thaw twice and stirred overnight at room temperature. After being evaporated, the suspension was poured into cold methanol. The blue precipitate was then filtered off and washed with hot methanol. The crude product was purified by column chromatography on silica gel using heptane/THF $(2: 1)$ as an eluent system and then recrystallized from $\mathrm{MeOH} / \mathrm{CH}_{2} \mathrm{Cl}_{2}$. The title compound $\mathbf{6 b}$ was obtained as a blue solid $(69 \mathrm{mg}$, 82\% yield). ${ }^{1}$ H NMR (400 MHz, THF- $\left.d_{8}\right): \delta=9.44-9.13(\mathrm{~m}, 8 \mathrm{H}), 8.44-8.29(\mathrm{~m}, 4 \mathrm{H}), 8.12$ - $7.98(\mathrm{~m}, 8 \mathrm{H}), 7.90-7.84(\mathrm{~m}, 4 \mathrm{H}), 7.81-7.67$ (m, 24H), 7.67 - $7.58(\mathrm{~m}, 8 \mathrm{H}), 7.46-7.38(\mathrm{~m}$, $8 \mathrm{H}), 7.36-7.27(\mathrm{~m}, 16 \mathrm{H}), 2.22-1.97(\mathrm{~m}, 32 \mathrm{H}), 1.22-1.05(\mathrm{~m}, 32 \mathrm{H}), 0.76-0.52(\mathrm{~m}, 80 \mathrm{H})$. ${ }^{13}$ C NMR (101 MHz, THF- $\left.d_{8}\right): \delta=151.3,151.1,142.4,140.8,134.2,131.2,127.9,127.2$, 126.4, 125.2, 125.1, 123.1, 121.6, 120.4, 120.1, 92.4, 92.3, 88.2, 55.4, 40.5, 26.4, 23.4, 13.6.

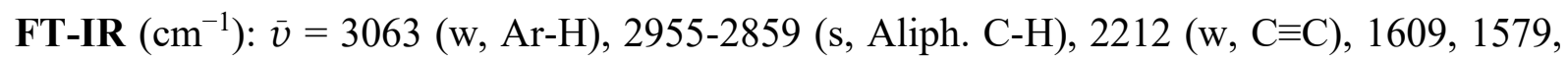
1489, 1466, 1453, 1379, 1308, 1154, 1137, 1096, 1050, 980, 894, 876, 830, 776, 739. HRMSMALDI (m/z): [M+H $]^{+}\left(\mathrm{C}_{248} \mathrm{H}_{225} \mathrm{~N}_{8} \mathrm{Zn}\right) 3378.753$ (calcd: 3378.7138). Anal. Calcd. (\%) for $\mathrm{C}_{248} \mathrm{H}_{224} \mathrm{~N}_{8} \mathrm{Zn} . \mathrm{CH}_{2} \mathrm{Cl}_{2}$ : C, 86.27; H, 6.57; N, 3.23. Found: C, 86.12; H, 6.33; N, 3.29.

General Procedure for Phthalocyanine 6a (G0): Phthalocyanine 6c (G): In a Schlenk tube, to a mixture of tetraiodophthalocyanine $\left(\mathbf{Z n I}_{4} \mathbf{P c}\right)(10 \mathrm{mg}, 0.009 \mathrm{mmol}, 1 \mathrm{eq}), \mathbf{8 c}(83 \mathrm{mg}$, $0.055 \mathrm{mmol}, 6 \mathrm{eq}), \mathrm{Pd}\left(\mathrm{PPh}_{3}\right)_{2} \mathrm{Cl}_{2}\left(0.06 \mathrm{mg}, 9 \times 10^{-5} \mathrm{mmol}, 1 \%\right.$ eq $)$ and $\mathrm{CuI}\left(0.01 \mathrm{mg}, 5.4 \times 10^{-}\right.$ ${ }^{5}$ mmol, $0.6 \%$ eq) were added THF ( $\left.3 \mathrm{~mL}\right)$ and $\mathrm{NEt}_{3}(3 \mathrm{~mL})$ under argon. Then, the system was degassed by freeze-pump-thaw twice and stirred overnight at room temperature. After being evaporated, the suspension was poured into cold methanol. The blue precipitate was then filtered off and washed with hot methanol. The crude product was purified by column chromatography on silica gel using heptane/THF $(3: 1)$ as an eluent system and then recrystallized from $\mathrm{MeOH} / \mathrm{CH}_{2} \mathrm{Cl}_{2}$. Complex 6c was obtained as a blue solid (46 mg, 76\% yield). ${ }^{1}$ H NMR (400 MHz, THF- $\left.d_{8}\right): \delta=9.80-9.21(\mathrm{~m}, 8 \mathrm{H}), 8.53-8.34(\mathrm{~m}, 4 \mathrm{H}), 8.16-7.98$ $(\mathrm{m}, 8 \mathrm{H}), 7.94-7.60(\mathrm{~m}, 76 \mathrm{H}), 7.59-7.51(\mathrm{~m}, 16 \mathrm{H}), 7.42-7.35(\mathrm{~m}, 16 \mathrm{H}), 7.34-7.24(\mathrm{~m}$, $32 \mathrm{H}), 2.20-1.93(\mathrm{~m}, 64 \mathrm{H}), 1.17-1.02(\mathrm{~m}, 64 \mathrm{H}), 0.74-0.48(\mathrm{~m}, 160 \mathrm{H}) .{ }^{13} \mathbf{C}$ NMR $(101 \mathrm{MHz}$, THF- $\left.d_{8}\right): \delta=151.2,151.1,142.4,142.2,140.8,134.5,131.2,131.1,127.9,127.2,126.3,125.1$, $125.0,124.5,124.1,123.1,121.5,121.4,120.4,120.0,92.3,88.0,87.9,55.3,40.4,26.323 .3$, 13.6. FT-IR $\left(\mathrm{cm}^{-1}\right): \bar{v}=3064$ (w, Ar-H), 2955-2859 (s, Aliph. C-H), 2210 (w, C $\left.\equiv \mathrm{C}\right), 1609$, 
1584, 1489, 1466, 1453, 1378, 1306, 1156, 1143, 1097, 1051, 893, 877, 830, 776, 739. HRMSMALDI (m/z): [M+H] ${ }^{+}\left(\mathrm{C}_{496} \mathrm{H}_{449} \mathrm{~N}_{8} \mathrm{Zn}\right) 6586.389$ (calcd: 6586.482). Anal. Calcd. (\%) for $\mathrm{C}_{496} \mathrm{H}_{448} \mathrm{~N}_{8} \mathrm{Zn}:$ C, 90.45; H, 6.86; N, 1.70. Found: C, 89.88; H, 6.72; N, 1.73.

General Procedure for Phthalocyanine 6a (G0): Phthalocyanine 7: In a Schlenk tube, to a mixture of tetraiodophthalocyanine $\left(\mathbf{Z n I}_{4} \mathbf{P c}\right)(27 \mathrm{mg}, 0.025 \mathrm{mmol}, 1 \mathrm{eq}), 9$ (60 $\mathrm{mg}, 0.15$ mmol, 6 eq $), \mathrm{Pd}\left(\mathrm{PPh}_{3}\right)_{2} \mathrm{Cl}_{2}\left(0.18 \mathrm{mg}, 2.5 \times 10^{-4} \mathrm{mmol}, 1 \%\right.$ eq $)$ and $\mathrm{CuI}\left(0.029 \mathrm{mg}, 1.5 \times 10^{-4}\right.$ mmol, $0.6 \%$ eq) were added THF $(5 \mathrm{~mL})$ and $\mathrm{NEt}_{3}(5 \mathrm{~mL})$ under argon. Then, the system was degassed by freeze-pump-thaw twice and stirred overnight at room temperature. After being evaporated, the suspension was poured into cold methanol. The dark green precipitate was then filtered off and washed with hot methanol. The crude product was purified by column chromatography on silica gel using heptane/THF $(2: 1)$ as an eluent system and then recrystallized from $\mathrm{MeOH} / \mathrm{CH}_{2} \mathrm{Cl}_{2}$. The title compound 7 was obtained as a green solid (48 $\mathrm{mg}$, $88 \%$ yield). ${ }^{1} \mathbf{H}$ NMR (400 MHz, THF- $\left.d 8\right): \delta=9.16-8.85(\mathrm{~m}, 8 \mathrm{H}), 8.26-8.12(\mathrm{~m}, 4 \mathrm{H}), 8.02$ - $7.86(\mathrm{~m}, 8 \mathrm{H}), 7.81-7.63(\mathrm{~m}, 20 \mathrm{H}), 7.62-7.57(\mathrm{~m}, 4 \mathrm{H}), 7.46-7.40(\mathrm{~m}, 4 \mathrm{H}), 7.38-7.30(\mathrm{~m}$, 8H), $2.22-1.99(\mathrm{~m}, 16 \mathrm{H}), 1.21-1.06(\mathrm{~m}, 16 \mathrm{H}), 0.76-0.52(\mathrm{~m}, 40 \mathrm{H}) .{ }^{13} \mathbf{C}$ NMR $(101 \mathrm{MHz}$, THF- $\left.d_{8}\right): \delta=152.4,152.2,151.4,151.3,142.4,141.1,138.3,137.6,132.5,132.4,132.2,131.5$, $128.1,127.4,126.4,126.1,124.3,124.2,124.0,123.3,122.9,122.1,120.6,120.4,93.3,93.2$, 91.5, 89.8, 55.6, 40.7, 23.6, 13.8. FT-IR $\left(\mathrm{cm}^{-1}\right): \bar{v}=3063$ (w, Ar-H), 2954-2858 (s, Aliph. CH), 2206 (w, C $\equiv C), 1609,1510,1466,1451,1397,1320,1298,1268,1148,1091,1048,938$, 894, 831, 776, 743. HRMS-MALDI (m/z): [M] ${ }^{+}\left(\mathrm{C}_{156} \mathrm{H}_{128} \mathrm{~N}_{8} \mathrm{Zn}\right) 2176.855$ (calcd: 2176.955). Anal. Calcd. (\%) for $\mathrm{C}_{156} \mathrm{H}_{128} \mathrm{~N}_{8} \mathrm{Zn}$ : C, 85.94; H, 5.92; N, 5.14. Found: C, 85.19; H, 5.94; N, 5.11 .

Spectroscopic Measurements. All photophysical properties have been performed with freshly-prepared air-equilibrated solutions at room temperature (298 K). UV-Vis absorption spectra were recorded on a BIO-TEK instrument UVIKON XL spectrometer or on a Jasco V570 spectrophotometer in THF (HPLC grade). Steady-state fluorescence measurements were performed on dilute solutions $\left(\mathrm{ca} .10^{-6} \mathrm{M}\right.$, optical density $\left.<0.1\right)$ contained in standard $1 \mathrm{~cm}$ quartz cuvettes using an Edinburgh Instrument (FLS920) spectrometer in photon-counting mode. Fully corrected emission spectra were obtained, for each compound, after excitation at the wavelength of the absorption maximum, with $A \lambda_{\mathrm{ex}}<0.1$ to minimize internal absorption. ${ }^{[42]}$

Measurements of singlet oxygen quantum yields $\left(\Phi_{\Delta}\right)$. Measurements were performed on a Fluorolog-3 (Horiba Jobin Yvon), using a 450W Xenon lamp, with air-equilibrated solutions. The optical density of the reference and the sample solution were set equal to 0.15 at 
the excitation wavelength (maximum of the Soret band). The emission at $1272 \mathrm{~nm}$ was detected using a liquid nitrogen-cooled Ge-detector model (EO-817L). The emission spectra were corrected for the wavelength dependence of the lamp intensity and the excitation monochromator efficiency (excitation correction). Singlet oxygen quantum yields $\Phi_{\Delta}$ were determined in toluene/pyridine (99:1) v/v solutions, using zinc(II) phthalocyanine (ZnPc) in a toluene/pyridine (99:1) mixture as reference solution $\left(\Phi_{\Delta}[\mathrm{ZnPc}]=0.61\right)^{[30]}$ and were estimated from corrected ${ }^{1} \mathrm{O}_{2}$ luminescence at $1272 \mathrm{~nm}$. The uncertainty of the values of the singlet oxygen quantum yields determined by this method was estimated to be \pm 0.05 .

Two-Photon Absorption Experiments. To span the $790-920 \mathrm{~nm}$ range, a Nd:YLFpumped Ti:sapphire oscillator (Chameleon Ultra, Coherent) was used generating 140 fs pulses at a $80 \mathrm{MHz}$ rate. The excitation power is controlled using neutral density filters of varying optical density mounted in a computer-controlled filter wheel. After five-fold expansion through two achromatic doublets, the laser beam is focused by a microscope objective $(10 \times$, NA 0.25 , Olympus, Japan) into a standard $1 \mathrm{~cm}$ absorption cuvette containing the sample. The applied average laser power arriving at the sample is typically between 0.5 and $40 \mathrm{~mW}$, leading to a time-averaged light flux in the focal volume on the order of $0.1-10 \mathrm{~mW} / \mathrm{mm}^{2}$. The fluorescence from the sample is collected in epifluorescence mode, through the microscope objective, and reflected by a dichroic mirror (Chroma Technology Corporation, USA; "red" filter set: $780 \mathrm{dxcrr}$ ). This makes it possible to avoid the inner filter effects related to the high dye concentrations used $\left(10^{-4} \mathrm{M}\right)$ by focusing the laser near the cuvette window. Residual excitation light is removed using a barrier filter (Chroma Technology; “'red"': e750sp-2p). The fluorescence is coupled into a $600 \mu \mathrm{m}$ multimode fiber by an achromatic doublet. The fiber is connected to a compact CCD-based spectrometer (BTC112-E, B\&WTek), which measures the two-photon excited emission spectrum. The emission spectra are corrected for the wavelengthdependence of the detection efficiency using correction factors established through the measurement of reference compounds having known fluorescence emission spectra. Briefly, the set-up allows for the recording of corrected fluorescence emission spectra under multiphoton excitation at variable excitation power and wavelength. 2PA cross sections $\left(\sigma_{2}\right)$ were determined from the two-photon excited fluorescence (TPEF) cross sections $\left(\sigma_{2} . \Phi_{\mathrm{F}}\right)$ and the fluorescence emission quantum yield $\left(\Phi_{\mathrm{F}}\right)$. TPEF cross sections of $10^{-4} \mathrm{M}$ THF solutions were measured relative to fluorescein in $0.01 \mathrm{M}$ aqueous $\mathrm{NaOH}$ using the well-established method described by $\mathrm{Xu}$ and $\mathrm{Webb}^{[43]}$ and the appropriate solvent-related refractive index 
corrections. ${ }^{[44]}$ The quadratic dependence of the fluorescence intensity on the excitation power was checked for each sample and all wavelengths.

DFT Computations and molecular dynamics simulations. Density functional theory ${ }^{[45]}$ calculations were performed with the hybrid Becke-3 parameter exchange functional ${ }^{[46]}$ and the Lee-Yang-Parr nonlocal correlation functional (B3LYP) ${ }^{[47]}$ implemented in the Gaussian 09 (Revision B.01) program suite ${ }^{[48]}$ using the pseudo-potentials LANL2DZ for zinc and the 6$31 \mathrm{G}^{*}$ basis set for $\mathrm{C}, \mathrm{H}, \mathrm{N}, \mathrm{O}$ with the default convergence criteria implemented in the program. The pseudo-potentials LANL2DZ were used for heavy atoms such as Zn atom. The UV-visible spectrum of $6 \mathbf{a}$ and 7 were simulated using TD-DFT calculations on 40 states. Molecular dynamics (MD) simulations on 6a-c and 7 have been performed at 298 K. Starting cubic cells ( $75 \times 75 \times 75 \AA$ ) containing one molecule of dendrimer and filled (or not) with THF molecules were constructed. After energy relaxation, the simulation consisted of a 4 ns isotherm at $25^{\circ} \mathrm{C}$ with the universal force field (NVT ensemble, $1 \mathrm{fs}$ time step, calculation time $\sim 20 \mathrm{~h}$ on 24 cores) under periodic boundary conditions. Calculations were carried out on the OCCIGEN calculator of the Centre Informatique National de l'Enseignement Supérieur (CINES, Montpellier, France) using the program suite Materials Studio (Forcite). Figures were generated with GaussView 5.0, and data were analyzed with GaussSum 2.2.6.1.

\section{ACKNOWLEDGEMENTS}

The authors acknowledge CNRS for their financial support and "the Ministère de l'Enseignement Supérieur et de la Recherche Scientifique de Tunisie" for $\mathrm{PhD}$ funding (SA and $\mathrm{SB})$ as well as China Scholarship Council (CSC) for PhD funding (ZS). This project was supported by the departmental committees CD35 and CD28 of the "Ligue contre le Cancer du Grand-Ouest”. We also thank Guillaume Clermont (ISM) for his help in the two-photon and singlet oxygen measurements. This work was granted access to the high-performance computing resources of CINES (Montpellier, France) under allocation 2020-A0080805032 awarded by GENCI.

\section{REFERENCES}

[1] E. Buhleier, W. Wehner, F. Voegtle, Synthesis 1978, 155-158.

[2] D. A. Tomalia, H. Baker, J. Dewald, M. Hall, G. Kallos, S. Martin, J. Roeck, J. Ryder, P. Smith, Polym. J. (Tokyo) 1985, 17, 117-132.

[3] G. R. Newkome, Z. Yao, G. R. Baker, V. K. Gupta, J. Org. Chem. 1985, 50, 2003-2004.

[4] C. J. Hawker, J. M. J. Frechet, J. Am. Chem. Soc. 1990, 112, 7638-7647. 
[5] A. W. Bosman, H. M. Janssen, E. W. Meijer, Chem. Rev. (Washington, D. C.) 1999, 99, $1665-1688$.

[6] a) M. Kozaki, A. Uetomo, S. Suzuki, K. Okada, Org. Lett. 2008, 10, 4477-4480; b) A. Uetomo, M. Kozaki, S. Suzuki, K.-I. Yamanaka, O. Ito, K. Okada, J. Am. Chem. Soc. 2011, 133, 13276-13279.

[7] a) J. N. G. Pillow, M. Halim, J. M. Lupton, P. L. Burn, I. D. W. Samuel, Macromolecules 1999, 32, 5985-5993; b) E. M. Harth, S. Hecht, B. Helms, E. E. Malmstrom, J. M. J. Fréchet, C. J. Hawker, J. Am. Chem. Soc. 2002, 124, 3926-3938; c) B. Li, K. Xu, M. Sun, Y. Fu, G. Yu, Y. Liu, Z. Bo, Macromolecules 2006, 39, 456-461; d) B. Li, J. Li, Y. Fu, Z. Bo, J. Am. Chem. Soc. 2004, 126, 3430-3431; e) M. Kozaki, K. Akita, S. Suzuki, K. Okada, Org. Lett. 2007, 9, 3315-3318.

[8] a) M. A. Oar, J. M. Serin, W. R. Dichtel, J. M. J. Frechet, T. Y. Ohulchanskyy, P. N. Prasad, Chem. Mater. 2005, 17, 2267-2275; b) M. A. Oar, W. R. Dichtel, J. M. Serin, J. M. J. Frechet, J. E. Roger, J.E. Slagla, P. A. Fleiz, L. S. Tan, T. Y. Ohulchanskyy, P. N. Prasad, Chem. Mater. 2006, 18, 3682-3692.

[9] a) O. Mongin, M. Sankar, M. Charlot, Y. Mir, M. Blanchard-Desce, Tetrahedron Lett. 2013, 54, 6474-6478; bO. Mongin, V. Hugues, M. Blanchard-Desce, A. Merhi, S. Drouet, D. Yao, C. Paul-Roth, Chem. Phys. Lett. 2015, 625, 151-156.

[10] a) C. O. Paul-Roth, J. A. G. Williams, J. Letessier, G. Simonneaux, Tetrahedron Lett. 2007, 48, 4317-4322; b) S. Drouet, C. O. Paul-Roth, Tetrahedron 2009, 65, 1069310700 ; c) S. Drouet, C. O. Paul-Roth, G. Simonneaux, Tetrahedron 2009, 65, $2975-$ 2981; d) S. Drouet, A. Merhi, G. Argouarch, F. Paul, O. Mongin, M. Blanchard-Desce, C. O. Paul-Roth, Tetrahedron 2012, 68, 98-105.

[11] a) D. Yao, V. Hugues, M. Blanchard-Desce, O. Mongin, C. O. Paul-Roth, F. Paul, New J. Chem. 2015, 39, 7730-7733; b) D. Yao, X. Zhang, O. Mongin, F. Paul, C. O. PaulRoth, Chem. Eur. J. 2016, 22, 5583-5597.

[12] L. B. Josefsen, R. W. Boyle, Theranostics 2012, 2, 916-966.

[13] a) Z. Sun, L.-P. Zhang, F. Wu, Y. Zhao, Adv. Funct. Mater. 2017, 27, 1704079; b) J. R. Starkey, A. K. Rebane, M. A. Drobizhev, F. Meng, A. Gong, A. Elliott, K. McInnerney, C. W. Spangler, Clin. Cancer Res. 2008, 14, 6564-6573; c) H. A. Collins, M. Khurana, E. H. Moriyama, A. Mariampillai, E. Dahlstedt, M. Balaz, M. K. Kuimova, M. Drobizhev, V. X. D. Yang, D. Phillips, A. Rebane, B. C. Wilson, H. L. Anderson, Nat. Photonics 2008, 2, 420-424; dM. Khurana, H. A. Collins, A. Karotki, H. L. Anderson, D. T. Cramb, B. C. Wilson, Photochem. Photobiol. 2007, 83, 1441-1448.

[14] a) P. Prabhu, V. Patravale, J. Biomed. Nanotechnol. 2012, 8, 859-882; bJ. Bhaumik, A. K. Mittal, A. Banerjee, Y. Chisti, U. C. Banerjee, Nano Res. 2015, 8, 1373-1394.

[15] L. Shi, C. Nguyen, M. Daurat, A. C. Dhieb, W. Smirani, M. Blanchard-Desce, M. GaryBobo, O. Mongin, C. Paul-Roth, F. Paul, Chem. Commun. 2019, 55, 12231-12234.

[16] a) M. Kimura, K. Nakada, Y. Yamaguchi, K. Hanabusa, H. Shirai, N. Kobayashi, Chem. Commun. 1997, 1215-1216; bM. Kimura, Y. Sugihara, T. Muto, K. Hanabusa, H. Shirai, N. Kobayashi, Chem. - Eur. J. 1999, 5, 3495-3500.

[17] a) J. Leclaire, Y. Coppel, A.-M. Caminade, J.-P. Majoral, J. Am. Chem. Soc. 2004, 126, 2304-2305; b) J. Leclaire, R. Dagiral, S. Fery-Forgues, Y. Coppel, B. Donnadieu, A.M. Caminade, J.-P. Majoral, J. Am. Chem. Soc. 2005, 127, 15762-15770; c) J. Leclaire, R. Dagiral, A. Pla-Quintana, A.-M. Caminade, J.-P. Majoral, Eur. J. Inorg. Chem. 2007, 2890-2896; d) M. Brewis, G. J. Clarkson, M. Helliwell, A. M. Holder, N. B. McKeown, Chem. - Eur. J. 2000, 6, 4630-4636; e) M. Brewis, M. Helliwell, N. B. McKeown, Tetrahedron 2003, 59, 3863-3872; f) M. Brewis, M. Helliwell, N. B. McKeown, S. Reynolds, A. Shawcross, Tetrahedron Lett. 2001, 42, 813-816; g) A. C. H. Ng, X.-y. Li, D. K. P. Ng, Macromolecules 1999, 32, 5292-5298; h) K. Kasuga, N. Matsuura, K.

\section{ACCEPTED MANUSCRIPT}


Inoue, M. Handa, T. Sugimori, K. Isa, M. Nakata, Chem. Lett. 2002, 352-353; i) C. A. Kernag, D. V. McGrath, Chem. Commun. 2003, 1048-1049; j) R. Ruiz-Gonzalez, F. Setaro, O. Gulias, M. Agut, U. Hahn, T. Torres, S. Nonell, Org. Biomol. Chem. 2017, 15, 9008-9017.

[18] S. Abid, S. Ben Hassine, N. Richy, F. Camerel, B. Jamoussi, M. Blanchard-Desce, O. Mongin, F. Paul, C. O. Paul-Roth, Molecules 2020, 25, 239.

[19] a) F. Figueira, P. M. R. Pereira, S. Silva, J. A. S. Cavaleiro, J. P. C. Tomé, Current Org. Synth. 2014, 11, 110-126; b) J. M. Dabrowski, B. Pucelik, A. Regiel-Futyra, M. Brindell, O. Mazuryk, A. Kyzioł, G. Stochel, W. Macyk, L. G. Arnaut, Coord. Chem. Rev. 2016, 325, 67-101; c) Y. Zhang, J. F. Lovell, Y. Zhang, Wiley Interdiscip Rev Nanomed Nanobiotechnol 2018, 9, 1-24; d) R. C. H. Wong, P.-C. Lo, D. K. P. Ng, Coord. Chem. Rev. 2019, 379, 30-46; e) J. Qiu, A. Hameau, J.-P. Majoral, A.-M. Caminade, J. Qiu, A. Hameau, J.-P. Majoral, A.-M. Caminade, J. Qiu, X. Shi, S. Mignani, S. Mignani, Chempluschem 2019, 84, 1070-1080.

[20] X. Zhang, S. Abid, L. Shi, Z. Sun, O. Mongin, M. Blanchard-Desce, F. Paul, C. O. PaulRoth, Dyes Pigm. 2018, 153, 248-255.

[21] E. M. Maya, P. Haisch, P. Vazquez, T. Torres, Tetrahedron 1998, 54, 4397-4404.

[22] D. A. Fernandez, J. Awruch, L. E. Dicelio, Photochem. Photobiol. 1996, 63, 784-792.

[23] a) C. Piechocki, J. Simon, J. Chem. Soc. Chem. Commun. 1985, 259-260; b) M. J. Chen, J. W. Rathke, J. Porph. Phthalocyan. 2001, 5, 528-536.

[24] A. de la Escosura, M. V. Martínez-Díaz, P. Thordarson, A. E. Rowan, R. J. M. Nolte, T. Torres, J. Am. Chem. Soc. 2003, 125, 12300-12308.

[25] L. Edwards, M. Gouterman, J. Mol. Spectro. 1970, 33, 292-310.

[26] A. Ogunsipe, D. Maree, T. Nyokong, J. Mol. Struct. 2003, 650, 131-140.

[27] A. Merhi, S. Drouet, N. Kérisit, C. O. Paul-Roth, Tetrahedron 2012, 68, 7901-.

[28] L. D. Lavis, R. T. Raines, ACS Chem. Bio. 2008, 3, 142-155

[29] P. S. Vincett, E. M. Voigt, K. E. Rieckhoff, J. Chem. Phys. 1971, 55, 4131-4140.

[30] a) M. van Leeuwen, A. Beeby, S. H. Ashworth, Photochem. Photobiol. Sci. 2010, 9, 370-375; b) M. van Leeuwen, A. Beeby, I. Fernandes, S. H. Ashworth, Photochem. Photobiol. Sci. 2014, 13, 62-69.

[31] N. Venkatram, D. N. Rao, L. Giribabu, S. V. Rao, Chem. Phys. Lett. 2008, 464, 211 215.

[32] M. Drobizhev, N. S. Makarov, A. Rebane, G. de la Torre, T. Torres, J. Phys. Chem. C 2008, 112, 848-859.

[33] a) M. Drobizhev, S. Makarov, Y. Stepanenko, A. Rebane, J. Chem. Phys. 2006, 124, 224701; b) A. Rebane, N. S. Makarov, M. Drobizhev, B. Spangler, E. S. Tarter, B. D. Reeves, C. W. Spangler, O. F. Meng, Z. Suo, J. Phys. Chem. C 2008, 112, 7997-8004.

[34] Z. Liu, X. Xiong, Y. Li, S. Lia, J. Qin, Photochem. Photobiol. Sci. 2011, 10, 1804-1809.

[35] H. M. Kim, B. R. Cho, Chem. Commun. 2009, 153-164.

[36] a) J. Zhao, D. Zhong, S. Zhou, Mater. Chem. B 2018, 6, 349-365; b) F. Ding, Y. Zhan, X. Lu, Y. Sun, Chem. Sci. 2018, 9, 4370-4380.

[37] a) M. G. Kuzyk, J. Chem. Phys. 2003, 119, 8327-8334; b) M. G. Kuzyk, J. Mater. Chem. 2009, 19, 7444-7465.

[38] a) J. F. Lovell, T. W. B. Liu, J. Chen, G. Zheng, Chemical Reviews 2018, 110, 28392857; b) F. Bolze, S. Jenni, A. Sour, V. Heitz, Chem. Commun. 2017, 53, 12857-12877.

[39] G. Ricciardi, A. Rosa, E. J. Baerends, J. Phys. Chem. A 2000, 105, 5242-5254.

[40] F. S. R. Ruiz-González, Ò. Gulías, U. H. M. Agut,T. Torres, S. Nonell, Org. Biomol. Chem. 2017, 15, 9008-9017.

[41] F. Malvolti, C. Rouxel, A. Triadon, G. Grelaud, N. Richy, O. Mongin, M. BlanchardDesce, L. Toupet, F. I. Abdul Razak, R. Stranger, M. Samoc, X. Yang, G. Wang, A. 
Barlow, M. P. Cifuentes, M. G. Humphrey, F. Paul, Organometallics 2015, 34, 54185437.

[42] a) N. Demas, G. A. Crosby, J. Phys. Chem. 1971 75, 991-1024; b) G. R. Eaton, S. S. Eaton, Acc. Chem. Res. 1988, 21, 107-113.

[43] C. Xu, W. W. Webb, J. Opt. Soc. Am. B 1996, 13, 481-491.

[44] M. H. V. Werts, N. Nerambourg, D. Pélégry, Y. Le Grand, M. Blanchard-Desce, Photochem. Photobiol. Sci. 2005, 4, 531-538.

[45] a) P. Hohenberg, W. Kohn, Phys. Rev. 1964, 136, B864-B871; b) R. G. Parr, W. Yang, Density-Functional Theory of Atoms and Molecules, Oxford University Press, Oxford, 1989.

[46] a) A. D. Becke, Phys. Rev. A 1988, 38, 3098-3100; b) A. D. Becke, J. Chem. Phys. 1993, 98, 1372-1377; c) A. D. Becke, J. Chem. Phys. 1993, 98, 5648-5652.

[47] C. Lee, W. Yang, R. G. Parr, Phys. Rev. B 1988, 37, 785-789.

[48] M. J. Frisch, G. W. Trucks, H. B. Schlegel, G. E. Scuseria, M. A. Robb, J. R. Cheeseman, G. Scalmani, V. Barone, B. Mennucci, G. A. Petersson, et al., Gaussian, Inc., Pittsburgh, PA, 2010. 